\title{
An Analysis of the EMV Channel Establishment Protocol
}

\author{
Christina Brzuska \\ School of Computer Science, \\ Tel Aviv University \\ brzuska@post.tau.ac.il \\ Bogdan Warinschi \\ Department of Computer \\ Science, University of Bristol \\ bogdan@cs.bris.ac.uk
}

\author{
Nigel P. Smart \\ Department of Computer \\ Science, University of Bristol \\ nigel@cs.bris.ac.uk \\ Gaven J. Watson \\ Department of Computer \\ Science, University of Bristol \\ gaven.watson@bristol.ac.uk
}

\begin{abstract}
With over 1.6 billion debit and credit cards in use worldwide, the EMV system (a.k.a. "Chip-and-PIN") has become one of the most important deployed cryptographic protocol suites. Recently, the EMV consortium has decided to upgrade the existing RSA based system with a new system relying on Elliptic Curve Cryptography (ECC). One of the central components of the new system is a protocol that enables a card to establish a secure channel with a card reader. In this paper we provide a security analysis of the proposed protocol, we propose minor changes/clarifications to the "Request for Comments" issued in Nov 2012, and demonstrate that the resulting protocol meets the intended security goals.

The structure of the protocol is one commonly encountered in practice: first run a key-exchange to establish a shared key (which performs authentication and key confirmation), only then use the channel to exchange application messages. Although common in practice, this structure takes the protocol out of the reach of most standard security models for key-exchange. Unfortunately, the only models that can cope with the above structure suffer from some drawbacks that make them unsuitable for our analysis. Our second contribution is to provide new security models for channel establishment protocols. Our models have a more inclusive syntax, are quite general, deal with a realistic notion of authentication (onesided authentication as required by EMV), and do not suffer from the drawbacks that we identify in prior models.
\end{abstract}

\section{Categories and Subject Descriptors}

C.2.0 [Computer-Communication Networks]: General—security and protection; K.6.5 [Management of Computing and Information Systems]: Security and Protection—authentication

\section{Keywords}

Key Exchange; Channel Establishment
Permission to make digital or hard copies of part or all of this work for personal or classroom use is granted without fee provided that copies are not made or distributed for profit or commercial advantage, and that copies bear this notice and the full citation on the first page. Copyrights for third-party components of this work must be honored. For all other uses, contact the owner/author(s). Copyright is held by the author/owner(s).

CCS'13, November 4-8, 2013, Berlin, Germany.

ACM 978-1-4503-2477-9/13/11.

http://dx.doi.org/10.1145/2508859.2516748.

\section{INTRODUCTION}

The EMV chip-and-pin system is used to secure a vast number of the world's credit card and ATM transactions and protects electronic banking in many countries [15]. The USA is due to switch from magnetic stripe to EMV cards from October 2015; after this time if a terminal (or ATM) does not support EMV then the merchant (ATM owner) will be liable for any fraudulent transactions and not the card issuer. The current EMV system uses RSA publickey cryptography, combined with DES and AES based symmetrickey cryptography. In the system, bank or credit card customers receive a plastic card containing an embedded chip that holds several cryptographic keys and can perform various cryptographic operations. The card is used to communicate with a terminal (typically a point-of-sale terminal in a shop, but other terminals are possible). In addition the card can produce cryptographically secured data which is sent to the cardholder and merchant's banks for processing. Nonetheless, the cryptographic functionality provided by the card in its first generation incarnation is relatively limited.

As part of a major reworking of the system, the EMV consortium has decided to replace RSA with ECC based systems and to let the card provide additional cryptographic functionalities. In November 2012 EMVCo released a Request-For-Comments [16] on a draft specification for an important sub-protocol within the system; namely a protocol that allows a card to establish a channel with a terminal. Calling the security of these protocols "important" is a serious understatement: the total number of public keys and certificates (1.62 billion [15]) deployed in the EMV systems dwarfs the paltry 5.8 million TLS certificates found in [11].

The problem of establishing and implementing secure channels is central to practical uses of cryptography and a superficial look at existing literature would lead one to believe that this is a solved problem. What can be simpler than first running a secure keyexchange protocol and then using the resulting keys to encrypt and authenticate the messages to be sent?

Indeed, there is a plethora of works looking at key establishment $[4,5,9]$ and a similar number of works treating secure channels based on shared keys [1, 6, 18]. Unfortunately, traditional key agreement models such as those following the schema set out by Bellare and Rogaway [4] have been shown to be less useful to analyze deployed protocols. In particular, they demand for session keys to be indistinguishable from a random string. However, whenever practical protocols implement explicit key confirmation, then key indistinguishability is violated.

Realizing that real-world protocols, such as TLS, are therefore outside the reach of the traditional models for key-exchange and channels, has triggered renewed interest in formal models for se- 
cure channels $[17,13,7]$. These approaches deal with what is essentially an overlap between the key-exchange part and the secure channel part of a channel-establishment protocol by either modifying the protocol, analyzing the overall protocol monolithically, or developing methods that allow for a modular analysis despite the overlapping phases.

The structure of the EMV protocol for establishing channels follows the recipe described above: during the key-exchange phase itself, the channel is already used before the deployed keys are accepted; and the messages that are sent over the channel are crucial for the security of the overall protocol. Our work can therefore be seen as a continuation of the recent thrust on research on models for channel establishment protocols. Below we describe the stateof-the art for such models, identify some of their weaknesses, and overview our results.

EXISTing Models for Secure Channel Establishment. When studying the combined properties of a key establishment and a secure channel protocol, one usually considers a security game where the execution model and the attack interface for key exchange and secure channels are "glued" together; and the adversary tries to break the secure channel using additional information that possibly leaked during the key-exchange phase. In particular, such a model also allows to analyze protocols that do not clearly separate the two components.

There are currently two approaches to prove the security in such a model. Very roughly, the first approach is to relax the security requirement on the keys by demanding that they are sufficiently strong to be used for the primitives that make-up the channel, and then show that the channel security relies only on these primitives. This modular approach is explored in [7], where a game-based composition theorem shows that a key-exchange protocol that is sufficiently strong for the primitives and a channel whose security reduces to these primitives automatically yield a secure protocol in the composed security model. The approach is shown to work for real-world protocols such as TLS.

In this paper, we prefer to avoid the machinery needed to work within this framework and instead concentrate on the approach of Jager et al. [13]. They propose to analyze channel establishment protocols, monolithically, with respect to a security model devised for this specific task. The models that they give are tailored for TLS and are not immediately applicable for EMV. Worse, both the original version of the model [13] and a more recent refined version [12] do not seem to appropriately capture the level of security that one would like. In brief, the former model is too strong, to the point that it actually rules as insecure protocols like TLS and the one that we analyze in this paper. The refined version, on the other hand seems to be too weak, as it takes away one of the adversary's abilities, an ability that reflects possible real-world powers.

A bit more in detail, the issue concerns the ability of an adversary to "reveal" a key, and its interaction with how "partnering" is defined. We explain these concepts next. Traditionally, reveal queries model the unintended leakage of session keys from a participant to assure that keys which leak from one session should not affect the security of other sessions. To distinguish between the same session and "other sessions", key exchange protocols match each session of a protocol with a single session of the intended partner via a partnering mechanism [3]. One of the first formulations of partnering relies on "matching" conversations [4] (the outgoing/incoming messages of one sessions are the same as the incoming/outgoing messages of its partner). The requirement is that if a session accepts, then it has had a matching conversation with "the right" partner. Unfortunately, in any protocol where some messages are sent encrypted with the key that is derived, the above requirement can- not hold. An attacker can proceed as follows. When an encrypted message goes on the network, block it, reveal the key that was used for encryption and then send to the recipient a different encryption of the same message, deploying fresh randomness. The two partners will not have a matching conversation, although the protocol will be executed successfully. In the full version of this paper [8] we describe an attack against entity authentication via matching conversations for TLS when an adversary is permitted to reveal keys as soon as they are derived. We stress that our attack uncovers subtleties in modelling and is not an actual attack on the TLS protocol. This attack demonstrates why neither version of ACCE is suitable for analysing these types of channel establishment protocol. The original ACCE model is too strong (the attack rules TLS insecure) and the revised too weak (the attack is no longer captured). Our model sits neatly in the middle, giving the adversary the capabilities of this attack but not treating it as an attack against the security of the protocol.

One approach to circumvent this attack is to preclude the adversary from performing such a reveal. This is the approach taken in [12] which only considers that keys can be revealed once the session in which they are derived had accepted. We find this restriction unsatisfactory. If reveals are considered possible, then they should be able to target a key as soon as that key has been derived. In particular, revealing a key that has just been used to perform an encryption should be allowed. A more in-depth discussion of weaknesses present in the existent models for channel-establishment protocols is given in the full version of this paper [8].

\section{Our Contribution}

In this paper we present a new definitional framework which addresses the problems identified in previous approaches. In particular we present a security model which is particularly tailored to the case of key-exchange followed by the creation of a secure channel. Our new framework is conceptually simpler than previous models and can be further extended to capture one-sided key agreement followed by composition with a secure channel. Providing a general model rather than one tailored specifically to EMV is important. A general framework can be used to analyse any channel establishment protocol and sets a clear goal that all such protocols should achieve. As a result, it is not only much easier to perform future analyses of such protocols but also facilitates comparison of different protocols which have now been proved secure with respect to the same notion of security. Below we highlight some of our contributions and techniques.

Models FOR CHANNEL-ESTABLISHMENT PROTOCOLS. For entity authentication, we deal with the realistic case of one-sided authentication. This is demanded by the protocol that we analyze: the card is authenticated, while the terminal is not. The EMV system is designed to authenticate the card holder. Intuitively, the only goal, a fraudulous terminal can pursue when interacting with a card, is to extract authentication data from the card in order to impersonate it later. As impersonation attacks are taken care of by secure onesided authenticated key agreement protocols already, one-sided authentication is considered a sufficient security goal in light of the costs that a certification structure for terminals would induce. We remark that existing models for channel-establishment concentrate on mutual authentication, and the case of one-sided authentication had been considered only sporadically in the key-exchange literature.

We also resolve the issue of bad interaction between partnering and reveal queries. Here, we take a different route than [13, 12]. Instead of weakening the adversary, we relax the partnering requirement: we only demand that partners agree on a common 
session identifier. This approach originates in the work of Bellare, Pointcheval, and Rogaway [3], is standard in key-exchange literature, and reflects an intuitively appealing level of security.

Finally we model and analyze unlinkability properties of the proposed protocol from EMVCo. One of the new design criteria of the protocol is a mild form of unlinkability; an adversary that sees a message flow between a terminal and a card should not be able to link this card's current transaction with a previous transaction from the same card. The new protocol aims to ensure this by using the channel to secure all traffic coming from the card. Thus any card identifying information (most importantly the certificate) will never be sent to the terminal in the clear. However the proposed protocol also uses a performance optimization in that the card uses a small ephemeral private key. We establish that using a small ephemeral key in this way should be avoided.

PRotocol ANALYSis \& EMV RECOMMENDATIONS. The EMV channel establishment protocol consists of a key exchange phase and an application phase. The key exchange phase is an ECCbased Diffie-Hellman-like protocol with one-sided authentication. We analyze the EMV channel establishment protocol and identify the assumptions under which it can be proved secure with respect to the notion that we put forth. We end this introduction by pointing out a number of recommendations related to the EMV protocol which have been passed to the designers as a result of our analysis:

1. The resulting Diffie-Hellman key should be hashed down to obtain the used symmetric keys. The proposal in [16] says to use a hash function or the $\mathrm{x}$-coordinate of the elliptic curve point as the key derivation function. The $\mathrm{x}$-coordinate of an elliptic curve point is a high-entropy value only. That is, it is not a random string, as required by primitives such as the ciphers that are deployed in the secure channel. We thus strongly recommend to extract the entropy via hashing and not use the x-coordinate directly.

2. The resulting keys should be used in a uni-directional manner; thus two keys need to be obtained from the hashing process. This avoids a large number of potential replay attacks on the application layer. If this was not done, the application layer would need to be implemented extremely carefully to thwart these attacks. Having two keys, one for each direction, makes the design of a secure application layer less vulnerable.

3. The card ephemeral key a should not be selected from the set $\{0,1\}^{32}$. We suggest that it is not restricted in size and instead chosen at random from $\mathbb{F}_{q}$. If the value $a$ is selected from the set $\{0,1\}^{32}$, then this has a significant effect on security. Not only does it reduce the scheme's ability to achieve unlinkablity. But in addition, when $a$ is selected from a small set an adversary could establish two sessions of one card which share the same key with a single terminal.

SCOPE The EMV standard has a huge scope, and not all of the next generation sub-protocols are currently fully defined. The goal of this paper is to analyse the proposed key exchange protocol according to the stipulated security goals while assuming as little as possible about the other protocols. For the secure channel, we have implicitly assumed, as this is not stated in [16], that the resulting secure channel should be secure against adversaries both deleting messages and playing messages out of order; since this is the usual definition of a secure channel. We did not assume any further security guarantees of the channel or other protocols; these need to be defined and proven separately from this paper.

\section{SCHEME}

Our presentation follows that in [16], augmented with information obtained from public discussions with the authors of the protocol at several meetings. The basic underlying idea of the protocol is to use a Diffie-Hellman key exchange in which one side (the card) has a static public key. In order to achieve unlinkability the certificate of this public key is not passed in the clear; instead, the card's static Diffie-Hellman key share is randomized by an additional ephemeral secret. The resulting Diffie-Hellman key is then hashed using a cryptographic hash function; which we will model as a random oracle.

The protocol uses a Diffie-Hellman group defined over an elliptic curve $G=E\left(\mathbb{F}_{p}\right)$ having group order a prime $q$ and also uses a base point $P \in G$. The prime $q$ is a function of an implicit security parameter $k$, but in practice the group is fixed and so all our results are given in the concrete security setting.

After the protocol has established secret keys, it uses them in a secure channel protocol (SendCh, ReceiveCh). On input an application message $m$ and state $s t_{e}$, SendCh returns a channel message $\mathfrak{c h}$. On input a channel message $\mathfrak{c h}$ and state $s t_{d}$, ReceiveCh returns an application message $m$. The secure channel protocol is based on a stateful authenticated-encryption (AE) scheme $A E=\{$ enc, dec $\}$. We assume that all plaintext headers used by the secure channel are unauthenticated, implying that no header is sent in clear as part of the AE scheme. The states $s t_{e}$ and $s t_{d}$ here model the fact that in practice sequence numbers are used to ensure that messages are delivered in order, thus the operations are stateful. We assume that the underlying AE scheme satisfies the properties of indistinguishability under chosen-ciphertext attack (IND-sfCCA) and integrity of plaintexts (INT-sfPTXT) for such stateful schemes, assuming the key-agreement scheme has generated a randomly distributed key. See Appendix A for formal definitions of these security notions.

We also assume that there is a public-key signature algorithm used to define certificates. In particular each card $C$ has a long term public/private key pair $\left(Q_{C}, d\right)$, where $d \in \mathbb{F}_{q}$ and $Q_{C}=d P \in G$. A certificate is a signature/message pair $\operatorname{cert}_{C}=\left(\operatorname{sig}_{\text {sk }}\left(Q_{C}\right), Q_{C}\right)$ provided by an issuing authority with a key pair (pk, sk) for some (unspecified) public-key signature algorithm (sig, ver). All that we require of the signature algorithm is that it be existentially unforgeable under a chosen-message attack (EUF-CMA). To save space we reserve a precise definition for this standard notion to the full version of our paper [8].

We are now in a position to define the EMV key establishment and secure channel protocol in Figure 1. As well as the components above, the protocol makes use of a hash function $\mathrm{H}$ that takes elements in the group $G$ and maps them onto a pair of keys for the $\mathrm{AE}$ scheme. The keys are used to secure the communication in both directions; we propose the use of two keys so that replay attacks are prevented at the level of the protocol as opposed to needing to be dealt with at the application layer.

As mentioned in the introduction the proposal by EMVCo [16] suggest that the ephemeral secret ${ }^{1} a$ should be small, (less than $2^{32}$ ). They state that this choice is "set to be fit for purpose for blinding a one-off session key". First note that the unlinkability property may be hard to achieve when $a$ is small: Given two public keys $Q_{1}$ and $Q_{2}$ and the first message of a session $a Q_{i}$, there is an obvious square-root attack which determines $Q_{i}$ when $a$ is small, i.e. an attack which runs in time roughly $2^{16}$ operations.

More seriously, the security of entity authentication would also be at risk. An adversary can perform an attack which allows two sessions of a card to share the same key with a single terminal.

\footnotetext{
${ }^{1}$ In the EMV draft $a$ is denoted $r$.
} 


\begin{tabular}{|c|c|c|}
\hline Card $(\mathbf{C})$ & & Terminal (T) \\
\hline \multirow{2}{*}{$a \stackrel{r}{\leftarrow} \mathbb{F}_{q}$} & $A=a Q_{C}$ & \\
\hline & $E=e P$ & $e \stackrel{r}{\leftarrow} \mathbb{F}_{q}$ \\
\hline$\left(\kappa_{e}^{C}, \kappa_{d}^{C}\right)=\mathrm{H}(d a E)$ & & $\left(\kappa_{d}^{T}, \kappa_{e}^{T}\right)=\mathrm{H}(e A)$ \\
\hline \multirow[t]{2}{*}{$\left(\mathfrak{c h}, s t_{e}^{C}\right)=\operatorname{SendCh}_{\kappa_{e}^{C}}\left(\operatorname{cert}_{C}\|a\| Q_{C} ; s t_{e}^{C}\right)$} & $\mathfrak{c h}$ & $\left(\operatorname{cert}_{C}\|a\| Q_{C}, s t_{d}^{T}\right)=\operatorname{ReceiveCh}_{\kappa_{d}^{T}}\left(\mathfrak{c h} ; s t_{d}^{T}\right)$ \\
\hline & & $\begin{array}{c}\text { Check } \operatorname{ver}_{\mathrm{pk}}\left(\operatorname{cert}_{C}, Q_{C}\right) \stackrel{?}{=} \text { true } \\
\text { Check } a Q_{C} \stackrel{?}{=} A\end{array}$ \\
\hline$\left(\mathfrak{c h}_{1}, s t_{e}^{C}\right)=\operatorname{SendCh}_{\kappa_{e}^{C}}\left(m_{1} ; s t_{e}^{C}\right)$ & $\mathfrak{c h}_{1}$ & $\left(m_{1}, s t_{d}^{T}\right)=$ ReceiveCh $_{\kappa_{d}^{T}}\left(\mathfrak{c h}_{1} ; s t_{d}^{T}\right)$ \\
\hline$\cdots$ & $\mathfrak{c h}_{2}$ & $\cdots$ \\
\hline$\left(m_{2}, s t_{d}^{C}\right)=$ ReceiveCh $_{\kappa_{d}^{C}}\left(\mathfrak{c h}_{2} ; s t_{d}^{C}\right)$ & & $\left(\mathfrak{c h}_{2}, s t_{e}^{T}\right)=\operatorname{SendCh}_{\kappa_{e}^{T}}\left(m_{2} ; s t_{e}^{T}\right)$ \\
\hline
\end{tabular}

Figure 1: Combined Authenticated Key Agreement Scheme and Secure Channel Protocol. Note that $\kappa_{e}^{C}=\kappa_{d}^{T}$ and $\kappa_{d}^{C}=\kappa_{e}^{T}$.

Consider a card with public key $Q=d P$ and assume that the ephemeral blinding exponents $a$ are small. In the first session the card chooses $a_{1}$ at random and sends $a_{1} Q$. The terminal will respond by choosing $e$ at random and returning $E=e P$. Their established key for this session will then be $\kappa=a_{1} d e P$. Next the card wants to establish a second session. It chooses at random a new ephemeral blind $a_{2}$ and sends $a_{2} Q$. When the ephemeral blinds $\left(a_{1}\right.$ and $\left.a_{2}\right)$ are small then an adversary can easily find $a^{\prime}=a_{1} / a_{2}$. The adversary can then calculate $a^{\prime} E$ and return this to the card. This second card session now establishes the key $a_{2} d\left(a^{\prime} E\right)=$ $a_{2} d\left(a_{1} / a_{2}\right) e P=a_{1} d e P=\kappa$; the same key as the first session. As a result of this (depending on the setting) the adversary may be able to perform a replay attack on the second card session.

There are other approaches which could prevent the latter attack (in cases where unlinkability is not an issue) but we believe increasing the size of $a$ to be the simplest and to offer the least chance of implementation errors being introduced. In the rest of the paper we assume that $a$ is chosen from $\mathbb{F}_{q}$ and therefore our security results apply only to this case.

\section{NEW SECURITY MODELS}

In this section we present our security models for the secure channel establishment and unlinkability. Most of the section is however devoted to the more complex case of modeling secure channel establishment.

PRELIMINARIES. Before giving our new definition we present some preliminary definitions. Let $I$ be the set of participants. Each participant has a distinct ID $i$, long-term public key $\mathrm{pk}_{i}$ and corresponding secret key $\mathrm{sk}_{i}$. The protocol description is defined by two efficiently computable stateful (sub)-protocols $P=\{\Pi, \mathcal{G}\}$. The protocol $\Pi$ defines how honest parties behave and $\mathcal{G}$ is a public/private key pair generation algorithm. Each execution of this algorithm maintains the following state information:

- $s t_{k} \in\{0,1\}^{*}$ is some state for the key exchange.

- $\delta \in\{$ derived, accept, reject, $\perp\}$ is current state of the key exchange (initialised to $\perp$ ).

- $\rho \in\{$ initiator, responder $\}$ is the role of the participant.

- sid a session identifier.
- pid a partner identifier

- $\kappa=\left(\kappa_{e}^{\rho}, \kappa_{d}^{\rho}\right) \in\left(\{0,1\}^{*} \cup\{\perp\}\right)^{2}$. This is the agreed pair of keys. The order of these keys depends on the role $\rho \in\{$ initiator, responder $\}$ and $\kappa=(\perp, \perp)$ unless $\delta=$ derived.

A Class of Protocols. In this paper we are concerned with the large class of protocols obtained by combining key-exchange with channel protocols, and where the two components cannot be easily disentangled. Existing models are either too specific [13] or do not consider the case of overlap between the components [7].

The syntax that we provide for such protocols naturally implies a classification of the different types of messages that may occur in an execution. The classification is a crucial ingredient of our security definitions. Specifically, we identify three different execution "modes" of protocols: establishing a key, sending, respectively receiving messages from the established channel. Formally, we define the honest operation of a participant by a triple $\Pi=($ KeyExch, SendCh, ReceiveCh).

Some of the messages sent during the key-exchange may travel over the channel so, strictly speaking, KeyExch may make use of the latter algorithms. To facilitate the description of the resulting complex interaction we define the algorithm EstChannel which, essentially, is in charge of establishing the channel. This algorithm may make calls to the algorithms defining $\Pi$. As an example, for EMV, one can view EstChannel as everything above the line drawn in Figure 1, see Figure 4 in Section 4 for full details. At any point during its execution protocol $\Pi$ takes as input a message $m$ and a message type, type $\in\{$ ap, ch $\}$ (indicating whether the message was received from the user's application or the channel, respectively), runs the appropriate algorithm, and returns the output of that algorithm.

We now detail the execution of $\Pi$ which is summarised in Figure 2. Prior to the channel being established (i.e. prior to $\delta=$ accept) whenever the input message $m$ has type ch then EstChannel will be called. This algorithm will make calls to KeyExch in order to establish the keys of the secure channel. The algorithm KeyExch takes as input a message $m$ and a state $s t_{k}$, and outputs a new message $m^{\prime}$ and updated state $s t_{k}$ (shared with EstChannel). The state $s t_{k}$ is used to manage the internal state of KeyExch and will contain the session identifier sid, partner identifier pid, the state of keys $\delta$, the established keys $\kappa_{e}, \kappa_{d}$, the states of the secure 
channel $s t_{e}, s t_{d}$, and any additional information $\alpha$. Before any keys have been derived $(\delta=\perp)$, EstChannel forwards messages directly to KeyExch. Once keys have been derived ( $\delta=$ derived) then we enter the key confirmation stage. At this point EstChannel initialises the states $s t_{e}, s t_{d}$ of the secure channel and makes appropriate $^{2}$ calls to SendCh and ReceiveCh before and after calling KeyExch. Finally, once KeyExch outputs $\delta=$ accept the secure channel has been successfully established.

After the channel has been established whenever the input message type is ch then ReceiveCh will be called. This models messages that are received from the channel (for decryption). It takes as input a message $m$ and state $s t_{d}$ and outputs a message $m^{\prime}$ for output to the user's application.

When the message type is ap then SendCh will be called. This models application messages that are input to be sent (encrypted) on the channel. It takes as input a message $m$ and state $s t_{e}$ and outputs a message $m^{\prime}$ for output to the channel. Note that if keys have not yet been established ( $\delta \neq$ accept) then such a call to SendCh will output $\perp$.

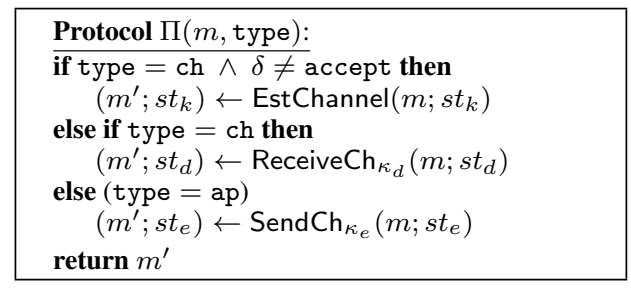

Figure 2: Honest Protocol Execution

ExECUTION MODEL. We consider the standard execution model for key exchange protocols where an adversary $\mathcal{A}$, is assumed to control all communication between participating parties i.e. the adversary can intercept all messages sent and inject any message that he wishes. Let $\Pi_{i}^{s}$ denote the oracle modelling participant $i \in I$ engaged in session $s$ of the protocol described above. Each oracle $\Pi_{i}^{s}$ runs the program $\Pi$ and maintains the states of that program instance. The adversary can make the following queries:

- NewSession $(i, \rho)$ : Create a session for user $i$ with role $\rho$.

- Send $\left(\Pi_{i}^{s}, m\right.$, type): Sends a message $m$ to $\Pi_{i}^{s}$ with type, type. As a result $\Pi_{i}^{s}$ will run $\Pi$ on input ( $m$, type).

- Reveal $\left(\Pi_{i}^{s}\right)$ : reveals the current session key $\kappa$ of $\Pi_{i}^{s}$.

- Corrupt $(i)$ : reveals the long-term private key of $i$.

PARTNERING AND FREShness. In order to define security for key-exchange protocols it is necessary to define the notion of partnering. Two participants should only establish a shared key if they have been successfully partnered. There are many approaches to defining such a notion. We begin by discussing the concept of matching conversations, introduced by Bellare and Rogaway [4] in the context of authenticated key exchange. A participant's conversation can be defined as a transcript of all the messages it receives and sends. As the name suggest, matching conversations defines two participants to be partnered if their transcripts match. It is this approach which is followed by Jager et al. [13] in their definition of ACCE. Unfortunately, when protocols use the session key to encrypt messages as part of a key-confirmation step, attacks

\footnotetext{
${ }^{2}$ Such calls will be dependent on the specific protocol construction.
}

may be possible which violate the requirements of matching conversations $^{3}$. For further details see the full version of this paper [8]. Notice however that while such attacks violate the matching conversation property, they should perhaps not be considered attacks. The plaintext that was sent by one party still reached its intended recipient. We interpret this attack as a limitation of the model: it may rule out as insecure protocols with no obvious weaknesses.

Our formulation is routed in the definition of partnering based on session identifiers by Bellare et al. [3]. Informally, Bellare et al. declared two oracles partnered if they have already derived keys and i) they both share the same session identifier sid, ii) they derived the same key $\kappa$, and iii) one oracle is an initiator and the other a responder. Moreover, to ensure each oracle accepted with only a single partner we also ask that iv) there should exist no other oracle which has derived keys and holds the same session identifier. Bellare et al. make a distinction between an oracle accepting and terminating. Accepting defines the event that the session keys have been established but the key confirmation steps are still to follow. An oracle terminates after the key confirmation steps have completed. Once keys are accepted they may be revealed but the key-exchange protocol has yet to terminate. We argue that a key is not "accepted" until after the key confirmation step since this step may fail. As a result, we use the terms derived and accepted, where derived corresponds to Bellare et al.'s accepted and our accepted corresponds to their terminated.

The specific formulation we give is similar but a stronger notion than that of Rogaway and Stegers [20]. First we define a notion of partnering which informally states that two oracles which have derived keys are partners if they share the same session identifier. The definition makes use of the following predicate on two oracles $\Pi_{i}^{s}$ and $\Pi_{j}^{t}$ holding $\left(\kappa_{i}^{s}, s i d_{i}^{s}, p i d_{i}^{s}\right)$ and $\left(\kappa_{j}^{t}, s i d_{j}^{t}, p i d_{j}^{t}\right)$, respectively:

$$
\begin{aligned}
& \mathrm{P}\left(\Pi_{i}^{s}, \Pi_{j}^{t}\right) \\
= & \begin{cases}\text { true } & \text { if } s i d_{i}^{s}=s i d_{j}^{t} \wedge \delta_{i}^{s}, \delta_{j}^{t} \in\{\text { derived, accept }\} \\
\text { false otherwise }\end{cases}
\end{aligned}
$$

Definition 1. (Partner) Two oracles $\Pi_{i}^{s}$ and $\Pi_{j}^{t}$ are said to be partnered if $\mathrm{P}\left(\Pi_{i}^{s}, \Pi_{j}^{t}\right)=$ true.

We follow this with three further definitions related to partnering which will be necessary when we give our main security definitions later. It shall be required that partnerings are valid, confirmed and unique. In short these three requirements ensure that any oracle that accepts, has a partner and that this partner is unique. More specifically, Valid means that partners will have corresponding partner identifiers (i.e. they both believe they are talking with each other), have different roles and share the same key. Confirmed partners ensures that for each oracle that accepts there exists at least one partner and for unique partners that there exists at most one.

Definition 2. (Valid Partners) A protocol $P=\{\Pi, \mathcal{G}\}$ ensures valid partners if the bad event notval does not occur, where notval is defined as follows:

$$
\begin{aligned}
& \exists \Pi_{i}^{s}, \Pi_{j}^{t} \text { such that: }\left(\mathrm{P}\left(\Pi_{i}^{s}, \Pi_{j}^{t}\right)=\text { true }\right) \\
& \quad \wedge\left(p i d_{i}^{s} \neq j \vee p i d_{j}^{t} \neq i \vee \rho_{i}^{s}=\rho_{j}^{t} \vee \kappa_{i}^{s} \neq \kappa_{j}^{t}\right) .
\end{aligned}
$$

Definition 3. (Confirmed Partners) A protocol $P=\{\Pi, \mathcal{G}\}$ ensures confirmed partners if the bad event notconf does not occur,

\footnotetext{
${ }^{3}$ The adversary reveals the key and then uses this to re-encrypt the confirmation message with new randomness. The two transcripts now differ for this message.
} 
where notconf is defined as follows:

$$
\begin{aligned}
& \exists \Pi_{i}^{s} \text { such that: } \delta_{i}^{s}=\text { accept } \\
& \quad \wedge i, p i d_{i}^{s} \text { not corrupt } \wedge\left(\mathrm{P}\left(\Pi_{i}^{s}, \Pi_{j}^{t}\right)=\text { false }: \forall \Pi_{j}^{t}\right) .
\end{aligned}
$$

Definition 4. (Unique Partners) A protocol $P=\{\Pi, \mathcal{G}\}$ ensures unique partners if the bad event notuni does not occur, where notuni is defined as follows:

$$
\begin{aligned}
& \exists \Pi_{i}^{s}, \Pi_{j}^{t}, \Pi_{k}^{r} \text { such that: }(j, t) \neq(k, r) \\
& \quad \wedge \mathrm{P}\left(\Pi_{i}^{s}, \Pi_{j}^{t}\right)=\text { true } \wedge \mathrm{P}\left(\Pi_{i}^{s}, \Pi_{k}^{r}\right)=\text { true. }
\end{aligned}
$$

A concept that plays a central role in defining security in twoparty protocols is that of "freshness". Intuitively, an oracle is fresh if it has accepted and an adversary had not "tampered" with it in any way, i.e. the adversary has not revealed or corrupted the oracle or its partner. This can be viewed as being related to the bad event notconf with the extra requirement that the oracle has not been revealed. A notion of freshness is necessary when defining security since the security guarantees are only for such oracles. The next definition formalises the concept. As with partnering we first introduced a predicate, $\mathrm{F}\left(\Pi_{i}^{s}\right)=$ true, if and only if:

1. $\delta_{i}^{s}=$ accept.

2. Oracle $\Pi_{i}^{s}$ has not been revealed and user $i$ is not corrupted.

3. No partner oracle of $\Pi_{i}^{s}$ has been revealed and no parent of such a oracle has been corrupted.

Definition 5. (Fresh) An oracle $\Pi_{i}^{s}$ is fresh if $\mathrm{F}\left(\Pi_{i}^{s}\right)=$ true.

\subsection{Security Definitions: Two-Sided Authen- tication Setting}

We formulate three levels of security: entity authentication, message authentication and message privacy. The later definitions rely on entity authentication and we start by defining that definition.

Entity AuthentiCATION. We consider that an adversary violates entity authentication if he can get a session to accept even if there is no unique session of its intended partner that has derived the same key. More formally, we wish to verify that none of the bad events notval, notconf, notuni occurs (cf. Definitions 2, 3, 4).

First consider the entity authentication experiment entauth that generates public/private key pairs for each user $i \in I$ (by running $\mathcal{G}$ ) and returns the public keys to $\mathcal{A}_{\text {ent }}$. The experiment then allows the adversary $\mathcal{A}_{\text {ent }}$ to make the queries NewSession $(i, \rho)$, $\operatorname{Reveal}\left(\Pi_{i}^{s}\right)$, Corrupt $(i)$ as well as $\operatorname{Send}\left(\Pi_{i}^{s}, m\right.$, type $)$ with operations type $\in\{$ ap, $\mathrm{ch}\}$. We say that an adversary violates entity authentication (and hence "wins" this experiment) if one of the bad events (notval, notconf, notuni) occurs. The adversary's advantage is defined to be:

$$
\operatorname{Adv}_{\Pi}^{\text {entauth }}\left(\mathcal{A}_{\text {ent }}\right)=\operatorname{Pr}[\text { notval } \vee \text { notconf } \vee \text { notuni }] .
$$

Definition 6. (Entity Authentication (EA)) Protocol $P=\{\Pi, \mathcal{G}\}$ is a $\left(t, \epsilon_{E A}\right)$-secure EA protocol if for all adversaries $\mathcal{A}_{\text {ent }}$ running in time at most $t, \mathbf{A d v}_{\Pi}^{\text {entauth }}\left(\mathcal{A}_{\text {ent }}\right) \leq \epsilon_{E A}$.

To define the security experiments for message authentication and privacy we shall make use of the following notation for lists maintained for each $\Pi_{i}^{s}$ as follows:

- Application messages sent Ap-S $\mathrm{S}_{i}^{s}$, i.e. the list of all messages $m$ input to Send $\left(\Pi_{i}^{s}, m\right.$, ap $)$.

- Channel messages sent $\mathrm{Ch}-\mathrm{S}_{i}^{s}$, i.e. the list of all outputs from Send $\left(\Pi_{i}^{s}, m\right.$, ap $)$.
- Channel messages received $\mathrm{Ch}-\mathrm{R}_{i}^{s}$, i.e. the list of all messages $m$ input to Send $\left(\Pi_{i}^{s}, m, \mathrm{ch}\right)$.

- Application messages received Ap- $\mathrm{R}_{i}^{s}$, i.e. the list of all outputs from Send $\left(\Pi_{i}^{s}, m, \mathrm{ch}\right)$.

As described at the beginning of this section during the execution of a protocol an oracle can receive two types of input, an application message (user input) or a channel message (received from the wire). Once a channel is established whenever an application message is input to Send, the protocol $\Pi$ is run and a channel message will be output and sent on the channel. Similarly whenever a channel message is input to Send, the protocol $\Pi$ is run and an application message will be output to the user. The above lists help us keep track of these messages and facilitate checks necessary in the following security models.

Message Authentication. We now turn our attention to message authentication. Here we wish to ensure the integrity and authenticity of all messages sent over the channel. For any two partner oracles $\Pi_{i}^{s}$ and $\Pi_{j}^{t}$, the oracle $\Pi_{i}^{s}$ should only successfully receive messages which were output by $\Pi_{j}^{t}$ and vice versa. In the definition which follows we formalise the intuition above by requiring that for any fresh oracle $\Pi_{i}^{s}$ with unique partner $\Pi_{j}^{t}$, the following holds $\operatorname{Prefix}\left(\mathrm{Ap}-\mathrm{R}_{i}^{s}, \mathrm{Ap}-\mathrm{S}_{j}^{t}\right)=$ true, where $\operatorname{Prefix}(X, Y)$ is the function which outputs true if $X$ is a prefix of $Y$ (provided not empty) and false otherwise. If this does not hold then the adversary was successfully fooled $\Pi_{i}^{s}$ into receiving an application message which was not output by the partnered oracle $\Pi_{j}^{t}$.

Consider the authentication experiment auth that generates public/private key pairs for each user $i \in I$ (by running $\mathcal{G}$ ) and returns the public keys to $\mathcal{A}$. The adversary is permitted to make the queries NewSession $(i, \rho)$, Reveal $\left(\Pi_{i}^{s}\right)$, Corrupt $(i)$ as well as Send $\left(\Pi_{i}^{s}, m\right.$, type $)$ with message type $\in\{$ ap, ch $\}$. On query Send $\left(\Pi_{i}^{s}, m\right.$, type $)$, the game behaves as in Figure 3(a).

For the session matching, we consider the notion of partnering as specified in Definition 1. The notion of freshness that we use in the following definition is according to Definition 5.

We define the following game $\operatorname{Exec}_{\Pi}^{\text {auth }}(\mathcal{A})$ between an adversary $\mathcal{A}$ and challenger $\mathcal{C}$ :

1. The challenger $\mathcal{C}$ generates public/private key pairs for each user $i \in I$ (by running $\mathcal{G}$ ) and returns the public keys to $\mathcal{A}{ }^{4}$

2. $\mathcal{A}$ is allowed to make as many NewSession, Reveal, Corrupt, Send queries as it likes.

3. The adversary stops with no output.

We say that an adversary $\mathcal{A}$ wins the game if there exists $\Pi_{i}^{s}$ with unique partner $\Pi_{j}^{t}$ such that $\mathrm{F}\left(\Pi_{i}^{s}\right)=$ true and the list $\mathrm{Ap}-\mathrm{R}_{i}^{s}$ is not a prefix of $\mathrm{Ap}-\mathrm{S}_{j}^{t}$.

We define the adversary's advantage as:

$$
\begin{gathered}
\operatorname{Adv}_{\Pi}^{\text {auth }}(\mathcal{A})=\operatorname{Pr}\left[\exists \Pi_{i}^{s}, \Pi_{j}^{t}: \mathrm{F}\left(\Pi_{i}^{s}\right)=\text { true } \wedge \mathrm{P}\left(\Pi_{i}^{s}, \Pi_{j}^{t}\right)=\right.\text { true } \\
\left.\wedge \operatorname{Prefix}\left(\mathrm{Ap}-\mathrm{R}_{i}^{s}, \mathrm{Ap}-\mathrm{S}_{j}^{t}\right)=\text { false }\right] .
\end{gathered}
$$

Definition 7. (Message Authentication (MA)) A protocol $P=$ $\{\Pi, \mathcal{G}\}$ is a $\left(t, \epsilon_{M A}\right)$-secure $\mathbf{M A}$ protocol if for all adversaries $\mathcal{A}_{\text {auth }}$ running in time at most $t, \operatorname{Adv}_{\Pi}^{\text {auth }}\left(\mathcal{A}_{\text {auth }}\right) \leq \epsilon_{M A}$.

Message Privacy. Next we consider the notion of message privacy. Our definition follows the standard indistinguishability

\footnotetext{
${ }^{4}$ Note that in the scheme considered in this paper, public keys of cards are not actually made public to $\mathcal{A}$ but are sent in encrypted form during the confirmation step.
} 


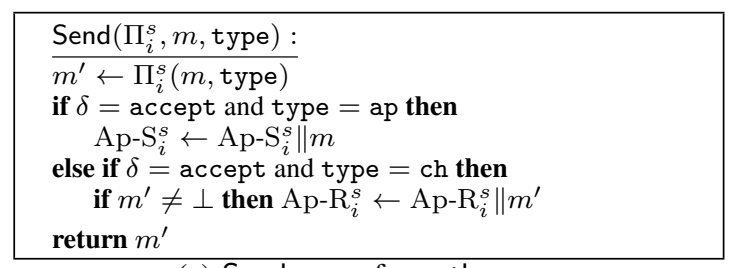

(a) Send query for auth game.

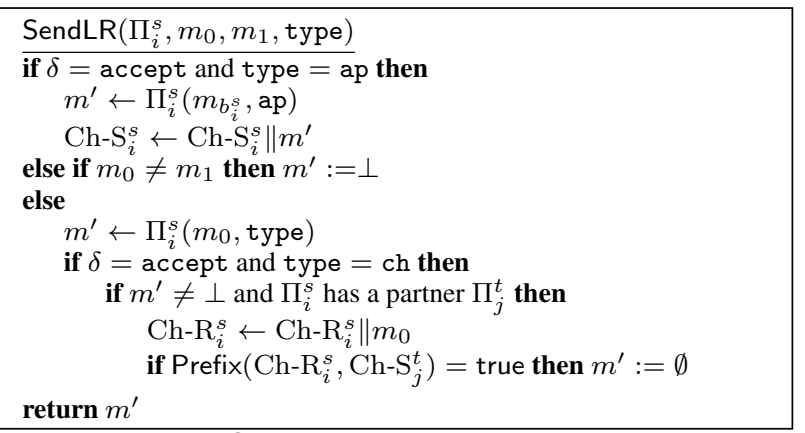

(b) SendLR query for priv game.

Figure 3: The Send (resp. SendLR) query for the auth (resp. priv) games

paradigm. The adversary should not be able to determine which set of message $\left\{m_{01}, m_{02}, m_{03}, \ldots\right\}$ and $\left\{m_{11}, m_{12}, m_{13}, \ldots\right\}$ has been transmitted on the secure channel.

The message privacy experiment priv initializes the states as in the authentication experiment auth, except that each session now also holds a random secret bit $b_{i}^{s}$. As before, the adversary can make the queries $\operatorname{NewSession}(i, \rho)$, Reveal $\left(\Pi_{i}^{s}\right)$, Corrupt $(i)$. In addition, we introduce a left-right version of Send $\left(\Pi_{i}^{s}, m\right.$, type $)$ which we use to model message privacy. Specifically, the query SendLR $\left(\Pi_{i}^{s}, m_{0}, m_{1}\right.$, type $)$ takes as input two messages and returns Send $\left(\Pi_{i}^{s}, m_{b_{i}^{s}}\right.$, type $)$. When type $\neq$ ap we require that these two message are equal,

$$
\operatorname{SendLR}\left(\Pi_{i}^{s}, m, m, \text { type }\right)=\operatorname{Send}\left(\Pi_{i}^{s}, m, \text { type }\right) .
$$

As before, two sessions are considered partners by Definition 1 . On the SendLR $\left(\Pi_{i}^{s}, m_{0}, m_{1}\right.$, type $)$ query, the game behaves as in Figure 3(b). We note that once the channel is establish, whenever $\operatorname{SendLR}\left(\Pi_{i}^{s}, m, m, \mathrm{ch}\right)$ is called (i.e. the input is a channel message), we allow the protocol to run as normal but check the lists $C R_{i}^{s}$ and $C S_{j}^{t}$. If the message $m$ was a channel output from $\Pi_{i}^{s}$ 's partner $\Pi_{j}^{t}$ then SendLR will not return anything. This allows the adversary to progress the state of an oracle but prevents them from trivially winning the game. The formulation is closely related to IND-sfCCA which we give in Appendix refapp:defn.

We define the following game $\operatorname{Exec}_{\Pi}^{\text {priv }}(\mathcal{A})$ between an adversary $\mathcal{A}$ and challenger $\mathcal{C}$ :

1. The challenger $\mathcal{C}$, generates public/private key pairs for each user $i \in I$ (by running $\mathcal{G}$ ) and returns the public keys to $\mathcal{A} .^{5}$

2. $\mathcal{A}$ is allowed to make as many NewSession, Reveal, Corrupt, SendLR queries as it likes.

3. Finally $\mathcal{A}$ outputs a tuple $\left(i, s, b^{\prime}\right)$.

We say the adversary $\mathcal{A}$ wins if its output $b^{\prime}=b_{i}^{s}$ and $\mathrm{F}\left(\Pi_{i}^{s}\right)=$ true (and has a unique partner) and the output of $\operatorname{Exec}_{\Pi}^{\text {priv }}(\mathcal{A})$ is set

\footnotetext{
${ }^{5}$ Note that in the scheme considered in this paper, public keys of cards are not actually made public to $\mathcal{A}$ but are sent in encrypted form during the confirmation step.
}

to 1 . Otherwise the output is 0 . Formally we define the advantage of $\mathcal{A}$ as

$$
\operatorname{Adv}_{\Pi}^{\text {priv }}(\mathcal{A})=\left|\operatorname{Pr}\left[\operatorname{Exec}_{\Pi}^{\text {priv }}(\mathcal{A})=1\right]-1 / 2\right| .
$$

Definition 8. (Message Privacy $(M P))$ A protocol $P=\{\Pi, \mathcal{G}\}$ is a $\left(t, \epsilon_{M P}\right)$-secure MP protocol if for all adversaries $\mathcal{A}_{\text {priv }}$ running in time at most $t, \mathbf{A d v}_{\Pi}^{\text {priv }}\left(\mathcal{A}_{\text {priv }}\right) \leq \epsilon_{M P}$.

We call a channel establishment protocol secure if it satisfies all of the three notions above. We call the resulting notion EAMAP for obvious reasons.

Definition 9. (EAMAP) Protocol $P=\{\Pi, \mathcal{G}\}$ is a $(t, \epsilon)$-secure EAMAP protocol if it is a $(t, \epsilon)$-secure EA protocol, a $(t, \epsilon)$-secure MA protocol and a $(t, \epsilon)$-secure MP protocol.

Remark 1. Our definitions are with respect to the specific type of protocol construction defined in Figure 2. We note however, that our notions can be extended to more general classes of protocols by simply placing fewer restrictions on the Send queries.

Remark 2. Our mechanism of defining message authentication by requiring that the list of messages received by a party is a prefix of the list of the messages sent by its partner is quite flexible. By appropriately modifying this requirement one can also capture more relaxed notions e.g. where packet dropping or reordering is allowed. Furthermore, we expect that with appropriate restrictions this mechanism can also be adapted to deal with fragmentation. This is a common feature of many secure/authenticated channels in practice and has been formally studied by Boldyreva et al. [6], but is not relevant for EMV.

\subsection{Security Definitions: One-Sided Authenti- cation Setting}

The above security definitions enforce mutual authentication, yet in many scenarios of practical concern only one party needs to be authenticated. For example, the protocol we consider requires authentication of the credit card but does not authenticate the communicating terminal. To model this situation we split our set of participants $I$ in two. Let $C$ be the set of authenticated participants (the cards) and let $T$ by the set of unauthenticated participants (the terminals), where unauthenticated participants do not hold a longterm private/public key pair. This formalisation is the same as that of registered and unregistered users in [17]. We say authenticated participants are always initiators and unauthenticated are always responders. As a result of this change we must alter our previous security definitions for entity authentication, message authentication, message privacy and their combination (EAMAP) to consider a one-sided protocol.

One-Sided Entity Authentication. In the one-sided setting a terminal $j \in T$ wishes to authenticate a card $i \in C$ and establish a key (additionally a secure channel) with this card. Since all $j \in T$ have no long-term secret then it would always be possible for an adversary to impersonate an unauthenticated participant and establish a session with a real card. We need only aim to ensure that a genuine card session is authenticated to an unauthenticated terminal.

We first describe informally the changes we must make to the original two-sided definitions. Recall the definition of partnering (cf. Definition 1 and the associated Definitions 2, 3 and 4). We are now only concerned with the case where a terminal is partnered correctly, i.e. for every terminal that accepts there exists a card session and this card session is unique. As a result this means we must 
give new one-sided versions of the definitions for valid partners and confirmed partners. These new definitions now specifically focus on the terminal, ensuring that it believes it is talking to the correct card and it is a responder (in the case of valid), and its partner is not corrupt (in the case of confirmed). Note that we do not adjust the uniqueness definition since we still wish to ensure that both a single card session cannot be partnered with two terminal sessions and a single terminal session cannot be partnered with two card sessions.

Definition 10. (One-Sided Valid Partners) Protocol $P=\{\Pi, \mathcal{G}\}$ ensures valid partners if the bad event os-notval does not occur, where os-notval is defined as follows:

$$
\begin{gathered}
\exists \Pi_{i}^{s}, \Pi_{j}^{t} \text { such that: } i \in T \wedge j \in C \wedge\left(\mathrm{P}\left(\Pi_{i}^{s}, \Pi_{j}^{t}\right)=\text { true }\right) \\
\wedge\left(p i d_{i}^{s} \neq j \vee \rho_{i}^{s} \neq \text { responder }=\rho_{j}^{t} \vee \kappa_{i}^{s} \neq \kappa_{j}^{t}\right) .
\end{gathered}
$$

Definition 11. (One-Sided Confirmed Partners) Protocol $P=$ $\{\Pi, \mathcal{G}\}$ ensures one-sided confirmed partners if os-notconf does not occur, where os-notconf is defined as follows:

$$
\begin{aligned}
& \exists \Pi_{i}^{s} \text { such that: } i \in T \wedge \delta_{i}^{s}=\text { accept } \\
& \quad \wedge \text { pid } d_{i}^{s} \text { not corrupt } \wedge\left(\mathrm{P}\left(\Pi_{i}^{s}, \Pi_{j}^{t}\right)=\text { false }: \forall \Pi_{j}^{t}\right) .
\end{aligned}
$$

We consider an adversary that violates one-sided entity authentication if he can get a terminal session to accept if there is no unique card session that has derived the same key. More formally, we define the os-entauth experiment in a similar fashion to before but now say that an adversary violates one-sided entity authentication (and hence "wins" this experiment) if one of the bad events (os-notval, os-notconf, notuni) occurs. The adversary's advantage is defined to be:

$$
\operatorname{Adv}_{\Pi}^{\text {os-entauth }}\left(\mathcal{A}_{\text {ent }}\right)=\operatorname{Pr}[\text { os-notval } \vee \text { os-notconf } \vee \text { notuni }] .
$$

Definition 12. (One-Sided EA (OS-EA)) A protocol $P=\{\Pi, \mathcal{G}\}$ is a $\left(t, \epsilon_{E A}\right)$-secure OS-EA protocol if for all adversaries $\mathcal{A}_{\text {ent }}$ running in time at most $t, \mathbf{A d v}_{\Pi}^{\text {os-entauth }}\left(\mathcal{A}_{\text {ent }}\right) \leq \epsilon_{E A}$.

One-Sided Message Authentication and PRivaCy. In order to adapt the definitions of message authenticity and privacy we must consider a one-sided version of freshness. The reason behind this again being that we wish to discount the trivial attack when the adversary impersonates an unauthenticated terminal $j \in T$. A card oracle is defined to be OS-fresh if it has accepted, has not been revealed or corrupted and it is partnered with a genuine terminal oracle. A terminal oracle is defined to be OS-fresh if it has accepted, has not been revealed and it is partnered with a card oracle that has not been revealed or corrupted. We formalise one-sided freshness as follows. As before we define a predicate, $\operatorname{OSF}\left(\Pi_{i}^{s}\right)=$ true, if and only if:

1. $\delta_{i}^{s}=$ accept.

2. Oracle $\Pi_{i}^{s}$ has not been revealed.

3. If $i \in C$ then it is uncorrupted and has a partner $\Pi_{j}^{t}$, where $j \in T$.

4. If $i \in T$ then $\Pi_{i}^{s}$ has a partner $\Pi_{j}^{t}$, where $j \in C$.

5. No oracle $\Pi_{j}^{t}$, such that $\mathrm{P}\left(\Pi_{i}^{s}, \Pi_{j}^{t}\right)=$ true, has been revealed and if $j \in C$ then it is uncorrupted.

Definition 13. (One-Sided Fresh) An oracle $\Pi_{i}^{s}$, is OS-fresh if $\operatorname{OSF}\left(\Pi_{i}^{s}\right)=$ true.
Using the above we can alter our previous experiments of auth and priv by requiring that the winning oracle is OS-fresh. We therefore obtain one-sided versions os-auth and os-priv, respectively.

Definition 14. (OS-MA/OS-MP) A protocol $P=\{\Pi, \mathcal{G}\}$ is a $(t, \epsilon)$-secure OS-MA protocol (or OS-MP resp.) if for all adversaries $\mathcal{A}$ running in time at most $t, \operatorname{Adv}_{\Pi}^{\text {os-auth }}(\mathcal{A}) \leq \epsilon$ (or $\operatorname{Adv}_{\Pi}^{\text {os-priv }}(\mathcal{A}) \leq \epsilon$ resp.).

We call a channel establishment protocol with one-sided authentication secure if it satisfies all three of the notions above.

Definition 15. (OS-EAMAP) A protocol $P=\{\Pi, \mathcal{G}\}$ is a $(t, \epsilon)$ secure OS-EAMAP protocol if it is a $(t, \epsilon)$-secure OS-EA protocol, a $(t, \epsilon)$-secure OS-MA protocol and a $(t, \epsilon)$-secure OS-MP protocol.

\subsection{Security Definitions: Unlinkability}

A further property that the EMVCo protocol aims to achieve is unlinkability. This means that it should be hard for an adversary to determine when two particular sessions involve the same card. Goldberg et al. [10] define a related notion of anonymity and unlinkability. They aim to prove a scheme secure if an authenticated party remains anonymous to its unauthenticated partner and hence call this internal anonymity. Here we are concerned with eavesdroppers external to the execution and hence define a new notion for external unlinkability.

We define this security property in terms of a game between adversary $\mathcal{A}$ and challenger $\mathcal{C}, \operatorname{Exec}_{\Pi}^{\text {unlink }}(\mathcal{A})$. Informally, the adversary is able to interact with the card and terminal much as in the key agreement game. At some point the adversary halts the first part of his game, and outputs two card identities on which it wishes to be challenged. The challenger then picks one of these two identities and passes to the adversary new oracles (i.e. card/terminal session) with respect to the chosen identity. The adversary can then make additional queries, bar Reveal or Corrupt queries on the two test oracles. At the end of the experiment the adversary needs to output which identity the challenger selected. More formally the game is defined as follows:

1. The challenger $\mathcal{C}$, generates public/private key pairs for each user $i \in C$ (by running $\mathcal{G}$ ) and returns the public keys to $\mathcal{A}^{6}$.

2. $\mathcal{A}$ is allowed to make as many NewSession, Reveal, Corrupt, Send queries as it likes.

3. At some point $\mathcal{A}$ outputs two identities $i_{0} \in C$ and $i_{1} \in C$.

4. The challenger then chooses a bit $b \stackrel{r}{\leftarrow}\{0,1\}$ and creates new oracles $\mathcal{O}_{C}=\Pi_{i_{b}}^{s}$ and $\mathcal{O}_{T}=\Pi_{j}^{t}$, (for some $j \in T$ ), by calling NewSession.

5. $\mathcal{A}$ continues making queries NewSession, Reveal, Corrupt, Send. However, $\mathcal{A}$ is allowed to query oracles $\mathcal{O}_{C}$ and $\mathcal{O}_{T}$ only with the Send query.

6. Eventually $\mathcal{A}$ stops and outputs a bit $b^{\prime}$.

We say the adversary $\mathcal{A}$ wins if its output $b^{\prime}=b$ and $\mathrm{P}\left(\mathcal{O}_{C}, \mathcal{O}_{T}\right)=$ true. In this case the output of $\operatorname{Exec}_{\Pi}^{\text {unlink }}(\mathcal{A})$ is set to one, otherwise the output is zero. We define the advantage of $\mathcal{A}$ to be

$$
\operatorname{Adv}_{\Pi}^{\text {unlink }}(\mathcal{A})=\left|\operatorname{Pr}\left[\operatorname{Exec}_{\Pi}^{\text {unlink }}(\mathcal{A})=1\right]-1 / 2\right| .
$$

Definition 16. (Unlinkability) A protocol $(\Pi, \mathcal{G})$ is $\left(t, \epsilon_{\text {unlink }}\right)$ unlinkable, if for all $\mathcal{A}$ running in time $t, \operatorname{Adv}_{\Pi}^{\text {unlink }}(\mathcal{A}) \leq \epsilon_{\text {unlink. }}$.

\footnotetext{
${ }^{6}$ Note that in the scheme considered in this paper, public keys of cards are not actually made public to $\mathcal{A}$ but are sent in encrypted form during the confirmation step.
} 


\section{SECURITY ANALYSIS}

In this section we state our main security results, and in particular clarify the assumptions under which the proposed EMV channelestablishment protocol is secure, namely the security of the signature scheme that is used to produce the certificates and, the Gap-DH and $\mathrm{CDH}$ assumptions in the group that underlies the scheme. We provide formal definitions of these assumptions later in this section.

Before stating our main security results we first give a formal description of the EMV protocol $P=\{\Pi, \mathcal{G}\}$. Here $\mathcal{G}$ is the keygeneration algorithm of the underlying signature scheme. As described at the start of Section 3, we define $\Pi$ in terms of a keyexchange protocol KeyExch and two algorithms which define the secure channel SendCh, ReceiveCh. In order to define channel establishment we utilise an additional algorithm EstChannel which makes calls to the underlying key exchange protocol KeyExch and secure channel algorithms SendCh, ReceiveCh. For simplicity we do not give a separate description of KeyExch but instead define EstChannel directly. We require three further algorithms which are used during the execution, loadkey, keyid, initial. An execution of a card oracle will involve a call to loadkey which will retrieve the public key $Q$, secret key $d$ and certificate cert associated to an identity $i$. Recall that $Q=d P$ and cert $=\left(\operatorname{sig}_{\text {sk }}(Q), Q\right)$. An execution of a terminal oracle will involve a call to keyid which will retrieve the identity $j$ associated to a public key $Q$. The algorithm initial will initialise the states of the secure channel $s t_{e}, s t_{d}$.

In Figure 4 we define the execution of EstChannel for a session $s$ of a party $i$ with state $s t_{k}=\left(\alpha, \delta, \rho, s t_{e}, s t_{d}, \kappa_{e}, \kappa_{d}, p i d, s i d\right)$ where each variable is initiated as $\perp$, the value $\alpha$ is used to store $a$ and $A$ for the card and the terminal respectively.

We now state our main security result.

THEOREM 1. If the Gap-DH problem is hard, the CDH problem is hard, $\mathrm{AE}=(\mathrm{enc}, \mathrm{dec})$ is an ind-sfcca secure and int-sfptxt secure AE scheme, and the signature scheme (sig, ver) used to produce card certificates is EUF-CMA, then the EMV protocol $P=$ $\{\Pi, \mathcal{G}\}$ in Figures 1 and 4 is secure in the sense of OS-EAMAP. In particular we have

- If there exists an adversary $\mathcal{A}$ running in time at most $t$ against the entity authentication property of OS-EAMAP security then there are adversaries $\mathcal{B}, \mathcal{C}, \mathcal{D}, \mathcal{E}$, such that

$$
\begin{aligned}
\operatorname{Adv}_{\Pi}^{\text {os-entauth }}(\mathcal{A}) \leq & \mathbf{A d v}_{(\text {sig,ver })}^{\text {eufcma }}(\mathcal{B}) \\
& +n_{C} \cdot(1-1 /|h|) \cdot \mathbf{A d v}_{E\left(\mathbb{F}_{p}\right)}^{\mathrm{Gap}-\mathrm{DH}}(\mathcal{C}) \\
& +n_{S} \cdot n_{C} \cdot \mathbf{A d v}_{\mathrm{AE}}^{\mathrm{int}-0}(\mathcal{D}) \\
& +n_{C}^{2} \cdot \mathbf{A d v}_{E\left(\mathbb{F}_{p}\right)}^{\mathrm{CDH}}(\mathcal{E}),
\end{aligned}
$$

where $\mathcal{B}, \mathcal{C}, \mathcal{D}, \mathcal{E}$ each run in time $t+O(\mu)$ where $\mu$ is total number of bits queried.

- If there exists an adversary $\mathcal{A}$ running in time at most $t$ against the message authentication property of OS-EAMAP security then there are adversaries $\mathcal{B}, \mathcal{C}$ and $\mathcal{D}$, such that

$$
\begin{aligned}
\operatorname{Adv}_{\Pi}^{\text {os-auth }}(\mathcal{A}) \leq & n_{S} \cdot\left(n_{C}+n_{T}\right) \cdot \mathbf{A d v}_{\mathrm{AE}}^{\text {intsptxt }}(\mathcal{D}) \\
& +n_{C} \cdot(1-1 /|h|) \cdot \mathbf{A d v}_{E\left(\mathbb{F}_{p}\right)}^{\text {Gap-DH }}(\mathcal{C}) \\
& +\mathbf{A d v}_{\Pi}^{\text {os-entauth }}(\mathcal{B}),
\end{aligned}
$$

where $\mathcal{B}$ runs in time $t$ and, $\mathcal{C}$ and $\mathcal{D}$ each run in time $t+$ $O(\mu)$ where $\mu$ is total number of bits queried.

- If there exists an adversary $\mathcal{A}$ against the message privacy property of OS-EAMAP security then there are adversaries

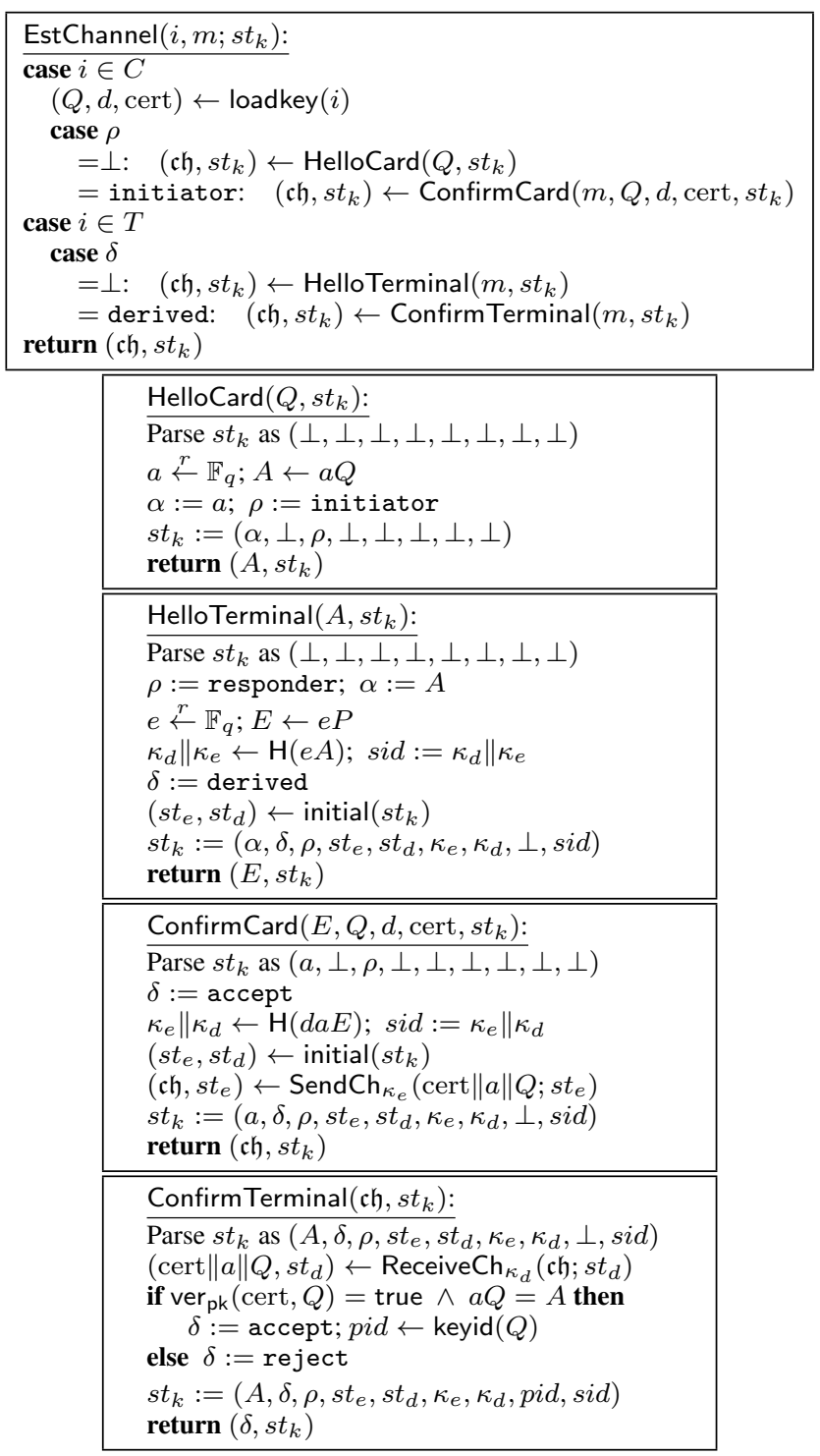

Figure 4: Channel Establishment Protocol EstChannel for EMV.

$$
\begin{aligned}
& \mathcal{B}, \mathcal{C} \text { and } \mathcal{D} \text {, such that } \\
& \mathbf{A d v}_{\Pi}^{\text {os-priv }}(\mathcal{A}) \leq n_{S} \cdot\left(n_{C}+n_{T}\right) \cdot \mathbf{A d v}_{\mathrm{AE}}^{\text {indsfcca }}(\mathcal{D}) \\
& +n_{C} \cdot(1-1 /|h|) \cdot \mathbf{A d v}_{E\left(\mathbb{F}_{p}\right)}^{\mathrm{Gap}-\mathrm{DH}}(\mathcal{C}) \\
& +\operatorname{Adv}_{\Pi}^{\text {os-entauth }}(\mathcal{B}) \text {, }
\end{aligned}
$$

where $\mathcal{B}$ runs in time $t$ and, $\mathcal{C}$ and $\mathcal{D}$ each run in time $t+$ $O(\mu)$ where $\mu$ is total number of bits queried.

where $n_{C}$ is the number of cards in the system, $n_{T}$ the number of terminals, $n_{S}$ the number of sessions and $|h|$ is the output size of the hash function.

Note that int- 0 defines security for an adversary against intsfptxt (or intsfctxt) that is permitted no encryption oracle queries.

We now prove each of the entity authentication, message authentication and message privacy properties in turn.

Before proceeding with the main proof we first examine a related concept of Key Secrecy for a simpler protocol, $\pi$, described in Fig- 
ure 5. To analyse this protocol we are only interested in whether the secret key remains secret, and so we introduce a new security game to model this fact. The security definition for Weak Key Secrecy can in found in Appendix B.

\begin{tabular}{|ccc|}
\hline Card (C) & & Terminal (T) \\
\hline$a \stackrel{r}{\leftarrow} \mathbb{F}_{q}$ & $\stackrel{A=a Q_{C}}{E=e P}$ & \\
& $\longleftarrow \stackrel{r}{\longleftarrow} \mathbb{F}_{q}$ \\
$\kappa=\mathrm{H}(d a E)$ & & $\kappa=\mathrm{H}(e A)$ \\
\hline
\end{tabular}

Figure 5: Unauthenticated Key-Agreement Scheme

Given this definition we can now analyse the protocol in Figure 5. The proof relies on the following problem being hard.

Definition 17. (Gap Diffie-Hellman) Let $\mathcal{O}_{\mathrm{DDH}}$ be an oracle that solves the DDH problem in $G$, i.e. takes as input $r P, s P, t P \in G$, and outputs one if $t P=r s P$ and zero otherwise.

The Gap Diffie-Hellman problem then asks that given $a P, b P \in G$ where $a, b \stackrel{r}{\leftarrow} \mathbb{F}_{q}$, and access to $\mathcal{O}_{\mathrm{DDH}}$, compute $a b P$ (i.e. solve $\mathrm{CDH})$. The advantage of an adversary $\mathcal{A}$ against the Gap DiffieHellman problem is defined by

$$
\operatorname{Adv}_{G}^{\mathrm{Gap}-\mathrm{DH}}(\mathcal{A})=\operatorname{Pr}\left[a, b \stackrel{r}{\leftarrow} \mathbb{F}_{q}: \mathcal{A}^{\mathcal{O}_{\mathrm{DDH}}}(a P, b P)=a b P\right] .
$$

LEMMA 1. The weak key secrecy of the reduced protocol $\pi$ is reducible to the Gap Diffie-Hellman assumption, i.e. we have for all adversaries $\mathcal{A}$ there exists an adversary $\mathcal{B}$ such that

$$
\mathbf{A d v}_{\pi}^{\mathrm{wKSec}}(\mathcal{A}) \leq n_{C} \cdot(1-1 /|h|) \cdot \mathbf{A d v}_{E\left(\mathbb{F}_{p}\right)}^{\mathrm{Gap}-\mathrm{DH}}(\mathcal{B}),
$$

where $n_{C}$ is the number of cards in the system and $|h|$ is the output length of the hash function.

The proof of this lemma uses a technique first presented by Kudla and Paterson [14] for analysing a hashed Diffie-Hellman based key agreement protocol. Our proof can be found in the full version of this paper [8].

\subsection{One-sided Entity Authentication}

We now turn to proving the different properties in our main theorem, starting with one-sided entity authentication. We make use of the following definition.

Definition 18. (Computational Diffie-Hellman) The CDH problem then asks that given $r P, s P \in G$, where $r, s \stackrel{r}{\leftarrow} \mathbb{F}_{q}$, compute $r s P$. The advantage of an adversary $\mathcal{A}$ against the $\mathrm{CDH}$ problem is defined by

$$
\operatorname{Adv}_{G}^{\mathrm{CDH}}(\mathcal{A})=\operatorname{Pr}\left[r, s \stackrel{r}{\leftarrow} \mathbb{F}_{q}: \mathcal{A}(r P, s P)=r s P\right] .
$$

LEMMA 2. If the gap-DH and $\mathrm{CDH}$ problems are hard, $\mathrm{AE}=$ (enc, dec) is an int-O secure AE scheme, and the signature scheme (sig, ver) used to produce card certificates is EUF-CMA, then the $E M V$ protocol $P=\{\Pi, \mathcal{G}\}$ is secure in the sense of OS-EA. In particular, if there exists an adversary $\mathcal{A}$ against $P=\{\Pi, \mathcal{G}\}$ in the sense of os-entauth then there are adversaries $\mathcal{B}, \mathcal{C}, \mathcal{D}$ and $\mathcal{E}$ such that

$$
\begin{aligned}
\operatorname{Adv}_{\Pi}^{\text {os-entauth }}(\mathcal{A}) \leq & \mathbf{A d v}_{(\text {sig,ver })}^{\text {eufcma }}(\mathcal{B}) \\
& +n_{C} \cdot(1-1 /|h|) \cdot \mathbf{A d v}_{E\left(\mathbb{F}_{p}\right)}^{\mathrm{Gap}-\mathrm{DH}}(\mathcal{C}) \\
& +n_{S} \cdot n_{C} \cdot \mathbf{A d v}_{\mathrm{AE}}^{\text {int-0 }}(\mathcal{D}) \\
& +n_{C}^{2} \cdot \mathbf{A d v}_{E\left(\mathbb{F}_{p}\right)}^{\mathrm{CDH}}(\mathcal{E}),
\end{aligned}
$$

where $n_{C}$ is the number of cards in the system, $n_{S}$ the number of sessions and $|h|$ is the output size of the hash function.

Proof. Let $\mathcal{A}$ be an adversary that wins the os-entauth experiment, that is the adversary succeeds in getting one of the bad events os-notval, os-notconf, notuni to occur. Then using the inequality $\operatorname{Pr}[A \vee B] \leq \operatorname{Pr}[A]+\operatorname{Pr}[B \mid \neg A]$, we obtain:

$$
\begin{aligned}
& \operatorname{Adv}_{\Pi}^{\text {os-entauth }}(\mathcal{A}) \\
= & \operatorname{Pr}[\text { os-notval } \vee \text { os-notconf } \vee \text { notuni }] \\
\leq & \operatorname{Pr}[\text { os-notconf }]+\operatorname{Pr}[\text { os-notval } \vee \text { notuni } \mid \neg \text { os-notconf }] \\
\leq & \operatorname{Pr}[\text { os-notconf }]+\operatorname{Pr}[\text { notuni }] \\
& \quad+\operatorname{Pr}[\text { os-notval } \mid \neg \text { os-notconf } \wedge \neg \text { notuni }] .
\end{aligned}
$$

In the full version of the paper [8] we prove the following bounds:

$$
\begin{aligned}
& \operatorname{Pr}[\text { os-notconf }] \leq \mathbf{A d v}_{(\text {sig,ver })}^{\text {eufcma }}(\mathcal{B}) \\
&+n_{C} \cdot(1-1 /|h|) \cdot \mathbf{A d v}_{E\left(\mathbb{F}_{p}\right)}^{\text {Gap-DH }}(\mathcal{C}) \\
&+n_{S} \cdot n_{C} \cdot \mathbf{A d v}_{\mathrm{AE}}^{\text {int-0 }}(\mathcal{D}) . \\
& \operatorname{Pr}[\text { notuni }] \leq n_{C}^{2} \cdot \mathbf{A d v}_{E\left(\mathbb{F}_{p}\right)}^{\mathrm{CDH}}(\mathcal{E}) . \\
& \operatorname{Pr}[\text { os-notval } \mid \neg \text { os-notconf } \wedge \neg \text { notuni }]=0 .
\end{aligned}
$$

\subsection{One-sided Message Authentication}

We now turn to the message authentication property:

LEMMA 3. If the Gap-DH problem is hard in $E\left(\mathbb{F}_{p}\right), \mathrm{AE}=$ (enc, dec) is an int-sfptxt secure AE scheme and $P=\{\Pi, \mathcal{G}\}$ is secure in the sense of os-entauth, then $P=\{\Pi, \mathcal{G}\}$ is secure in the sense of os-auth. In particular if there is an adversary $\mathcal{A}$ against the os-auth property then there are adversaries $\mathcal{B}, \mathcal{C}$ and $\mathcal{D}$ such that

$$
\begin{aligned}
\operatorname{Adv}_{\Pi}^{\text {os-auth }}(\mathcal{A}) \leq & n_{S} \cdot\left(n_{C}+n_{T}\right) \cdot \mathbf{A d v}_{\mathrm{AE}}^{\text {intsfptxt }}(\mathcal{D}) \\
& +n_{C} \cdot(1-1 /|h|) \cdot \mathbf{A d v}_{E\left(\mathbb{F}_{p}\right)}^{\operatorname{Gap} \text {-DH }}(\mathcal{C}) \\
& +\mathbf{A d v}_{\Pi}^{\text {os-entauth }}(\mathcal{B}),
\end{aligned}
$$

where $n_{C}$ is the number of cards in the system, $n_{T}$ the number of terminals, $n_{S}$ the number of sessions and $|h|$ is the output size of the hash function.

Proof. We shall prove this result via a sequence of games. Let $\mathcal{A}$ be an adversary attacking $\Pi$ in the sense of auth.

Game 0: This game is identical to $\operatorname{Exec}_{\Pi}^{\text {os-auth }}(\mathcal{A})$.

$$
\operatorname{Pr}[\text { Game0 } \Rightarrow 1]=\operatorname{Adv}_{\Pi}^{\text {os-auth }}(\mathcal{A}) .
$$

Game 1: This proceeds identically to the previous game but aborts if a terminal $(i \in T)$ oracle $\Pi_{i}^{s}$ accepts but has no unique partner oracle which establishes the same key. It is easy to see that if this event occurs then one of the events os-notval, os-notconf, notuni has occurred. Therefore,

$$
\operatorname{Pr}[\text { Game } 0 \Rightarrow 1] \leq \operatorname{Pr}[\text { Game1 } \Rightarrow 1]+\operatorname{Adv}_{\Pi}^{\text {os-entauth }}(\mathcal{B})
$$

Game 2: This proceeds identically to the previous game but aborts if $\mathcal{A}$ makes a query to $\mathrm{H}$ which reveals the key for an oracle $\Pi_{i}^{s}$. Again it is easy to see that

$$
\operatorname{Pr}[\text { Game1 } \Rightarrow 1] \leq \operatorname{Pr}[\text { Game2 } \Rightarrow 1]+\operatorname{Adv}_{\pi}^{\mathrm{wKSec}}\left(\mathcal{C}^{\prime}\right) .
$$


Game 3: The challenger now selects at random an oracle $\Pi_{i^{*}}^{s^{*}}$. The game aborts if Prefix $\left(\operatorname{Ap}-\mathrm{R}_{i}^{s}, \operatorname{Ap}-\mathrm{S}_{j}^{t}\right)=$ false for $(i, s) \neq\left(i^{*}, s^{*}\right)$. Since $i^{*}$ is chosen at random from $I=C \cup T$ we have:

$$
\operatorname{Pr}[\text { Game2 } \Rightarrow 1] \leq n_{S} \cdot\left(n_{C}+n_{T}\right) \cdot \operatorname{Pr}[\text { Game3 } \Rightarrow 1] .
$$

It remains to study the probability that $\mathcal{A}$ wins (Game $3 \Rightarrow 1$ ). We shall use $\mathcal{A}$ in Game3 to construct a new adversary $\mathcal{D}$ against the INT-sfPTXT security of AE. What we effectively do is set the output of the random oracle $\mathrm{H}$ for the key corresponding to $\Pi_{i^{*}}^{s^{*}}$ to be the key chosen at random for the INT-sfPTXT experiment. The adversary $\mathcal{D}$ will use its enc and dec oracles to provide simulations of $\mathcal{A}$ 's Send queries with $\Pi_{i^{*}}^{s^{*}}$ or $\Pi_{j^{*}}^{t^{*}}\left(\right.$ where $\mathrm{P}\left(\Pi_{i^{*}}^{s^{*}}, \Pi_{j^{*}}^{t^{*}}\right)=$ true $)$ as the input session. All other queries NewSession, Reveal, Corrupt and all other Send queries will be simulated internally by $\mathcal{D}$. We further explain how Send queries for $\Pi_{i^{*}}^{s^{*}}$ or $\Pi_{j^{*}}^{t^{*}}$ are simulated. When $\mathcal{A}$ makes a Send query for $\Pi_{i^{*}}^{s^{*}}$ or $\Pi_{j^{*}}^{t^{*}}$ before $\delta=$ derived, $\mathcal{D}$ performs the key exchange himself by using appropriate randomness. Once $\delta=$ derived, $\mathcal{D}$ must make calls to enc and dec in order to perform the key confirmation. Since the confirmation message received/sent by $\Pi_{i^{*}}^{s^{*}}$ should be equal to that sent/received by the partnered card/terminal session respectively, when $\mathcal{D}$ calls dec he will not be given the plaintext due to restrictions on decryption queries in the INT-sfPTXT model. If this happens then he accepts the card session and sets $\delta=$ accept, otherwise he sets $\delta=$ reject. After $\delta=$ accept whenever $\mathcal{A}$ makes a Send query where type $=$ ap or ch, $\mathcal{D}$ calls his enc or dec oracle, respectively. Since $\mathcal{A}$ does not make any reveal queries or hash queries corresponding to the key of $\Pi_{i^{*}}^{s^{*}}$ the simulation remains perfect. If $\mathcal{A}$ wins the auth game then $\operatorname{Prefix}\left(\mathrm{Ap}-\mathrm{R}_{i^{*}}^{s^{*}}, \mathrm{Ap}-\mathrm{S}_{j^{*}}^{t^{*}}\right)=$ false and thus $\mathcal{A}$ has output a ciphertext forgery which allows $\mathcal{D}$ to win the INT-sfPTXT game.

$$
\operatorname{Pr}[\text { Game3 } \Rightarrow 1] \leq \operatorname{Adv}_{\mathrm{AE}}^{\text {intsfptxt }}(\mathcal{D}) .
$$

Combining all of the above we obtain

$$
\begin{aligned}
& \mathbf{A d v}_{\Pi}^{\text {os-auth }}(\mathcal{A}) \\
&= \operatorname{Pr}[\operatorname{Game} 0 \Rightarrow 1] \\
& \leq \operatorname{Pr}[\operatorname{Game} 1 \Rightarrow 1]+\mathbf{A d v}_{\Pi}^{\text {os-entauth }}(\mathcal{B}) \\
& \leq \operatorname{Pr}[\operatorname{Game} 2 \Rightarrow 1]+\mathbf{A d v}_{\pi}^{\text {wKSec }}\left(\mathcal{C}^{\prime}\right)+\mathbf{A d v}_{\Pi}^{\text {os-entauth }}(\mathcal{B}) \\
& \leq n_{S} \cdot\left(n_{C}+n_{T}\right) \cdot \operatorname{Pr}\left[\operatorname{Game}^{\text {of }} \Rightarrow 1\right]+\mathbf{A d v}_{\pi}^{\text {wKSec }}\left(\mathcal{C}^{\prime}\right) \\
& \quad+\mathbf{A d v}_{\Pi}^{\text {os-entauth }}(\mathcal{B}) \\
& \leq n_{S} \cdot\left(n_{C}+n_{T}\right) \cdot \mathbf{A d} \mathbf{d v}_{\mathrm{AE}}^{\text {intsfptxt }}(\mathcal{D})+\mathbf{A d v}_{\pi}^{\text {wKSec }}\left(\mathcal{C}^{\prime}\right) \\
& \quad+\mathbf{A d v}_{\Pi}^{\text {os-entauth }}(\mathcal{B})
\end{aligned}
$$

With the final result following from applying Lemmas 1 and 2.

\subsection{One-sided Message Privacy}

We now turn to the message privacy property:

LEMMA 4. If the Gap-DH problem is hard in $E\left(\mathbb{F}_{p}\right), \mathrm{AE}=$ (enc, dec) is an ind-sfcca secure AE scheme and $P=\{\Pi, \mathcal{G}\}$ is secure in the sense of os-entauth. Then $P=\{\Pi, \mathcal{G}\}$ is secure in the sense of os-priv, i.e. any adversary $\mathcal{A}$ against the os-priv property can be turned into adversaries $\mathcal{B}, \mathcal{C}$ and $\mathcal{D}$ such that

$$
\begin{aligned}
\operatorname{Adv}_{\Pi}^{\text {os-priv }}(\mathcal{A}) \leq & n_{S} \cdot\left(n_{C}+n_{T}\right) \cdot \mathbf{A d v}_{\mathrm{AE}}^{\text {indsfcca }}(\mathcal{D}) \\
& +n_{C} \cdot(1-1 /|h|) \cdot \mathbf{A d v}_{E\left(\mathbb{F}_{p}\right)}^{\mathrm{Gap}-\mathrm{DH}}(\mathcal{C}) \\
& +\mathbf{A d v}_{\Pi}^{\text {os-entauth }}(\mathcal{B})
\end{aligned}
$$

The proof of this is similar to Lemma 3; details can be found in the full version of this paper [8]. We again use game hopping with almost identical hops to the previous proof. The final step is to argue that security in Game 3 is reducible to IND-sfCCA security.

\subsection{Unlinkability}

Finally, we present our theorem for unlinkability:

THEOREM 2. If gap-DH is hard and $\mathrm{AE}=$ (enc, dec) is an ind-sfcca secure AE scheme, then $P=\{\Pi, \mathcal{G}\}$ is secure in the sense of unlink; in particular we have

$$
\begin{aligned}
& \operatorname{Adv}_{\Pi}^{\text {unlink }}(\mathcal{A}) \\
\leq & n_{C}^{2} \cdot\left(\operatorname{Adv}_{\mathrm{AE}}^{\text {indsfcca }}(\mathcal{C})+n_{C} \cdot(1-1 /|h|) \cdot \mathbf{A d v}_{E\left(\mathbb{F}_{p}\right)}^{\mathrm{Gap}-\mathrm{DH}}(\mathcal{B})\right)
\end{aligned}
$$

where, again, $n_{C}$ is the number of cards in the system and $|h|$ is the output size of the hash function.

Note that if $a$ were instead chosen to be of size $2^{32}$ (as suggested by the RFC) our security analysis would show only 16 bits of security. We refer the reader to the proof in Appendix $C$ for further details.

\section{ACKNOWLEDGEMENTS}

This work was support in part by ERC Advanced Grant ERC2010-AdG-267188-CRIPTO. The second author was also partially supported by a Royal Society Wolfson Merit Award. The first author was supported by the Israel Science Foundation (grant 1076/11 and 1155/11), the Israel Ministry of Science and Technology (grant 3-9094), and the German-Israeli Foundation for Scientific Research and Development (grant 1152/2011).

\section{REFERENCES}

[1] Mihir Bellare, Tadayoshi Kohno, and Chanathip Namprempre. Breaking and provably repairing the $\mathrm{SSH}$ authenticated encryption scheme: A case study of the encode-then-encrypt-and-mac paradigm. ACM Trans. Inf. Syst. Secur., 7(2):206-241, 2004.

[2] Mihir Bellare and Chanathip Namprempre. Authenticated encryption: Relations among notions and analysis of the generic composition paradigm. In Tatsuaki Okamoto, editor, ASIACRYPT, volume 1976 of Lecture Notes in Computer Science, pages 531-545. Springer, 2000.

[3] Mihir Bellare, David Pointcheval, and Phillip Rogaway. Authenticated key exchange secure against dictionary attacks. In Bart Preneel, editor, EUROCRYPT, volume 1807 of Lecture Notes in Computer Science, pages 139-155. Springer, 2000.

[4] Mihir Bellare and Phillip Rogaway. Entity authentication and key distribution. In Douglas R. Stinson, editor, CRYPTO, volume 773 of Lecture Notes in Computer Science, pages 232-249. Springer, 1993.

[5] Simon Blake-Wilson, Don Johnson, and Alfred Menezes. Key agreement protocols and their security analysis. In Michael Darnell, editor, IMA Int. Conf., volume 1355 of Lecture Notes in Computer Science, pages 30-45. Springer, 1997.

[6] Alexandra Boldyreva, Jean Paul Degabriele, Kenneth G. Paterson, and Martijn Stam. Security of symmetric encryption in the presence of ciphertext fragmentation. In David Pointcheval and Thomas Johansson, editors, EUROCRYPT, volume 7237 of Lecture Notes in Computer Science, pages 682-699. Springer, 2012. 
[7] Christina Brzuska, Marc Fischlin, Nigel P. Smart, Bogdan Warinschi, and Stephen C. Williams. Less is more: Relaxed yet composable security notions for key exchange. IACR Cryptology ePrint Archive, 2012:242, 2012.

[8] Christina Brzuska, Nigel P. Smart, Bogdan Warinschi, and Gaven J. Watson. An analysis of the EMV channel establishment protocol. IACR Cryptology ePrint Archive, 2013:31, 2013.

[9] Ran Canetti and Hugo Krawczyk. Analysis of key-exchange protocols and their use for building secure channels. In Birgit Pfitzmann, editor, EUROCRYPT, volume 2045 of Lecture Notes in Computer Science, pages 453-474. Springer, 2001.

[10] Ian Goldberg, Douglas Stebila, and Berkant Ustaoglu. Anonymity and one-way authentication in key exchange protocols. Designs, Codes and Cryptography, 2012. Online first; print version to appear.

[11] Nadia Heninger, Zakir Durumeric, Eric Wustrow, and J.Alex Halderman. Mining your Ps and Qs: Detection of widespread weak keys in network devices. In USENIX Security Symposium - 2012, pages 205-220, 2012.

[12] Tibor Jager, Florian Kohlar, Sven Schäge, and Jörg Schwenk. On the security of TLS-DHE in the standard model. IACR Cryptology ePrint Archive, 2011:219, 2011.

[13] Tibor Jager, Florian Kohlar, Sven Schäge, and Jörg Schwenk. On the security of TLS-DHE in the standard model. In Reihaneh Safavi-Naini and Ran Canetti, editors, CRYPTO, volume 7417 of Lecture Notes in Computer Science, pages 273-293. Springer, 2012.

[14] Caroline Kudla and Kenneth G. Paterson. Modular security proofs for key agreement protocols. In Bimal K. Roy, editor, ASIACRYPT, volume 3788 of Lecture Notes in Computer Science, pages 549-565. Springer, 2005.

[15] EMVCo LLC. EMV deployment statistics. http://www.emvco.com/about_emvco.aspx?id=202, 2012.

[16] EMVCo LLC. EMV ECC key establishment protocols. http://www.emvco.com/specifications.aspx?id=243, 2012.

[17] Paul Morrissey, Nigel P. Smart, and Bogdan Warinschi. The TLS handshake protocol: A modular analysis. J. Cryptology, 23(2):187-223, 2010.

[18] Kenneth G. Paterson, Thomas Ristenpart, and Thomas Shrimpton. Tag size does matter: Attacks and proofs for the tls record protocol. In Dong Hoon Lee and Xiaoyun Wang, editors, ASIACRYPT, volume 7073 of Lecture Notes in Computer Science, pages 372-389. Springer, 2011.

[19] John Pollard. Monte Carlo methods for index computation mod p. Mathematics of Computation, 32:918-924, 1978.

[20] Phillip Rogaway and Till Stegers. Authentication without elision: Partially specified protocols, associated data, and cryptographic models described by code. In CSF, pages 26-39. IEEE Computer Society, 2009.

\section{APPENDIX}

\section{A. BASIC SECURITY DEFINITIONS}

The underlying AE scheme we assume satisfies the following two properties which are variants of the stateful security models of Bellare et al. [1] and Paterson et al. [18].

An adversary against a stateful encryption scheme needs to be given the capability to progress the scheme's state without trivially winning the security experiment. It is for this reason that there is a subtle difference between the standard notions of Indistinguishability under Chosen-Ciphertext Attack (IND-CCA) and
Integrity of Plaintexts (INT-PTXT)[2], and their stateful versions IND-sfCCA and INT-sfPTXT. An adversary against IND-sfCCA and INT-sfPTXT security is permitted to query the decryption oracle with an output from the encryption oracle in order to progress the state but the output of this query should not be returned to the adversary (to avoid the trivial attack).

In order to match with our security definitions of auth and priv we alter previous IND-sfCCA of Bellare et al. [1] and introduce a new notion for INT-sfPTXT (which is analogous to the definition of INT-sfCTXT given by Bellare at al.) to now compare the lists $\mathrm{M}-\mathrm{E}$ and $\mathrm{C}-\mathrm{E}$ with $\mathrm{M}-\mathrm{D}$ and $\mathrm{C}-\mathrm{D}$ respectively. In the case of INDsfCCA, C-E is the list of all ciphertexts output by the encryption oracle and C-D is the list of all ciphertexts successfully decrypted by the decryption oracle. For INT-sfPTXT the lists M-E, M-D correspond to plaintext messages input to and output from the encryption and decryption oracle, respectively. In order to prevent an adversary trivially winning he is not permitted to see the output of the decryption oracle if C-D (M-D resp.) is a prefix of C-E (M-E resp.), i.e. if all ciphertexts (plaintexts resp.) decrypted so far were output by the encryption oracle in the case of IND-sfCCA or if all plaintexts output so far were inputs to the encryption oracle in the case of INT-sfPTXT.

Definition 19. (IND-sfCCA) Consider the scheme $\mathrm{AE}=\left\{\mathrm{enc}_{\kappa}\right.$, $\left.\operatorname{dec}_{\kappa}\right\}$. Let $\mathcal{A}$ be an adversary with access to a left-or-right encryption oracle enc $\kappa_{\kappa}\left(h, \mathrm{LR}_{b}\left(m_{0}, m_{1}\right) ; s t_{e}\right)$ and a decryption oracle $\operatorname{dec}_{\kappa}\left(h, c ; s t_{d}\right)$. It is mandated that any two messages queried to enc ${ }_{\kappa}\left(h, \mathrm{LR}_{b}\left(m_{0}, m_{1}\right) ; s t_{e}\right)$ have equal length. We define an experiment as follows:

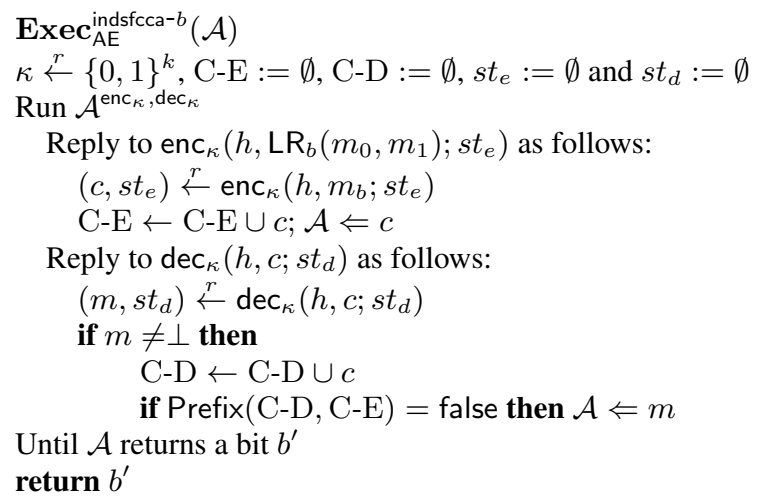

The attacker wins when $b^{\prime}=b$, and his advantage is defined as

$$
\operatorname{Adv}_{\mathrm{AE}}^{\text {indsfcca }}(\mathcal{A})=2 \operatorname{Pr}\left[\operatorname{Exec}_{\mathrm{AE}}^{\text {indsfcca-b }}(\mathcal{A})=b\right]-1 .
$$

Definition 20. (INT-sfPXT) Consider the scheme AE $=\left\{\right.$ enc $_{\kappa}$, $\left.\operatorname{dec}_{\kappa}\right\}$. Let $\mathcal{A}$ be an adversary with oracle access to enc $c_{\kappa}\left(h, m ; s t_{e}\right)$ and $\operatorname{dec}_{\kappa}\left(h, c ; s t_{d}\right)$. We define an experiment as follows: 


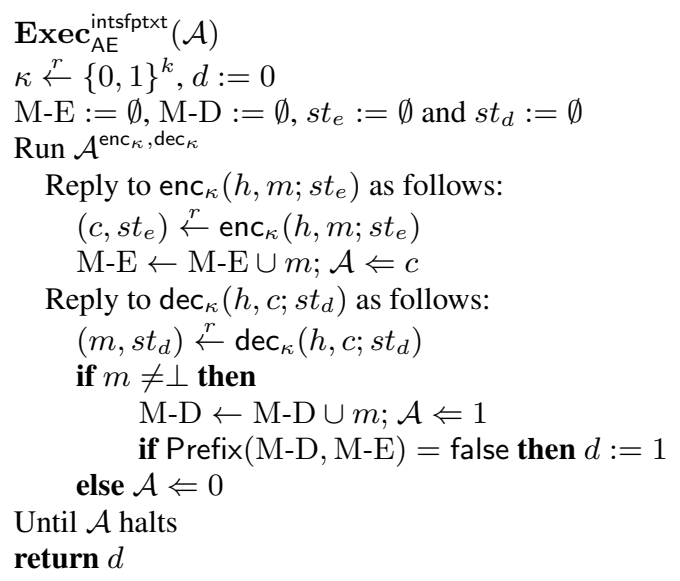

The advantage $\operatorname{Adv}_{\mathrm{AE}}^{\text {intsfptxt }}(\mathcal{A})$ of an adversary is defined as

$$
\operatorname{Adv}_{\mathrm{AE}}^{\text {intsfptxt }}(\mathcal{A})=\operatorname{Pr}\left[\operatorname{Exec}_{\mathrm{AE}}^{\text {intsfptxt }}(\mathcal{A})=1\right] .
$$

In addition we define the related notion int- 0 which considers an adversary $\mathcal{A}$ against intsfptxt (or in fact intsfctxt) that is permitted no encryption oracle queries.

\section{B. KEY SECRECY}

We define the following game $\operatorname{Exec}_{\Pi}^{\mathrm{KSec}}(\mathcal{A})$ between an adversary $\mathcal{A}$ and challenger $\mathcal{C}$ :

1. The challenger $\mathcal{C}$, generates public/secret key pairs for each user $i \in I$ (by running $\mathcal{G}$ ) and returns the public keys to $\mathcal{A}$.

2. Adversary $\mathcal{A}$ is allowed to make as many NewSession, Send, Reveal, Corrupt queries as it likes.

3. Finally $\mathcal{A}$ outputs a pair $\Pi^{*}$ and $\kappa^{*}$.

We say the adversary $\mathcal{A}$ wins if $\mathrm{F}\left(\Pi^{*}\right)=$ true and $\kappa^{*}$ is the key agreed by $\Pi^{*}$. In this case the output of $\operatorname{Exec}_{\Pi}^{\mathrm{KSec}}(\mathcal{A})$ is set to 1 . Otherwise the output is 0 . We define the advantage of $\mathcal{A}$ to be

$$
\operatorname{Adv}_{\Pi}^{\mathrm{KSec}}(\mathcal{A})=\left|\operatorname{Pr}\left[\operatorname{Exec}_{\Pi}^{\mathrm{KSec}}(\mathcal{A})=1\right]\right| .
$$

Definition 21. (Key Secrecy) $P=\{\Pi, \mathcal{G}\}$ is a $\left(t, \epsilon_{\mathrm{KSec}}\right)$-key secret $\mathbf{A K}$ protocol if for all adversaries $\mathcal{A}$ running in time $t$ the following holds:

1. In the presence of a benign adversary on $\Pi_{i}^{s}$ and $\Pi_{j}^{t}$ both oracles accept holding the same session identifier sid, the same session key $\kappa$, and this key is distributed uniformly at random on $\{0,1\}^{k}$.

2. $\mathcal{A}$ 's advantage is bounded by $\operatorname{Adv}_{\Pi}^{\mathrm{KSec}}(\mathcal{A}) \leq \epsilon_{\mathrm{KSec}}$.

We can also define a weaker version of this model for one-sided authentication by running the experiment in the same way as before but changing the winning condition slightly. We say the adversary $\mathcal{A}$ wins the wKSec experiment if $\operatorname{OSF}\left(\Pi^{*}\right)=$ true and $\kappa^{*}$ is the key agreed by $\Pi^{*}$.

Definition 22. (Weak Key Secrecy) $P=\{\Pi, \mathcal{G}\}$ is a $\left(t, \epsilon_{\mathrm{wKSec}}\right)$ weak Key-secure AK protocol if for all adversaries $\mathcal{A}$ running in time $t$ the following holds:

1. In the presence of a benign adversary on $\Pi_{i}^{s}$ and $\Pi_{j}^{t}$ both oracles accept holding the same session identifier sid, the same session key $\kappa$ and this key is distributed uniformly at random on $\{0,1\}^{k}$.

2. $\mathcal{A}$ 's advantage is bounded by $\operatorname{Adv}_{\Pi}^{\mathrm{wKSec}}(\mathcal{A}) \leq \epsilon_{\mathrm{wKSec}}$.

\section{PROOF OF THEOREM 2}

Proof. We shall prove this result via a sequence of games. Let $\mathcal{A}$ be an adversary attacking $\Pi$ in the sense of unlink. Game 0: This game is identical to $\operatorname{Exec}_{\Pi}^{\text {unlink }}(\mathcal{A})$.

$$
\operatorname{Pr}[\operatorname{Game} 0 \Rightarrow 1]-\frac{1}{2}=\operatorname{Adv}_{\Pi}^{\text {unlink }}(\mathcal{A}) \text {. }
$$

Game 1: The challenger now selects at random $i_{0}^{*}$ and $i_{1}^{*}$. The game aborts and returns random $b^{\prime}$ if $\mathcal{A}$ does not output $i_{0}=i_{0}^{*}$ and $i_{1}=i_{1}^{*}$. We obtain

$$
\operatorname{Pr}[\text { Game } 0 \Rightarrow 1]-\frac{1}{2} \leq n_{C}^{2} \cdot\left(\operatorname{Pr}[\text { Game } 1 \Rightarrow 1]-\frac{1}{2}\right) .
$$

Game 2: This proceeds identically to the previous game but aborts if $\mathcal{A}$ makes a query to $\mathrm{H}$ which reveals the key for the oracle $\mathcal{O}$. We obtain

$$
\operatorname{Pr}[\text { Game1 } \Rightarrow 1] \leq \operatorname{Pr}[\text { Game2 } \Rightarrow 1]+\operatorname{Adv}_{\pi}^{\mathrm{wKSec}}\left(\mathcal{B}^{\prime}\right) .
$$

Game 3: This proceeds identically to the previous game except that whenever Send is called with $\mathcal{O}_{C}$ and type $=$ ap then the challenger replaces $m$ with a random message which it then encrypts. Again it is easy to see that we obtain

$$
\operatorname{Pr}[\text { Game2 } \Rightarrow 1] \leq \operatorname{Pr}[\operatorname{Game} 3 \Rightarrow 1]+\operatorname{Adv}_{\mathrm{AE}}^{\text {indsfcca }}(\mathcal{C}) .
$$

It remains to study the probability that $\mathcal{A}$ wins (Game2 $\Rightarrow 1$ ). Since ciphertexts are now distributed uniformly at random the only useful information that $\mathcal{A}$ can determine are the public keys $Q_{i_{0}^{*}}$, $Q_{i_{1}^{*}}$, and the blinded challenge value $a Q_{i_{b}^{*}}$. Since $a$ is chosen at random from $\mathbb{F}_{q}$, then the distributions $\left(Q_{i_{0}^{*}}, Q_{i_{1}^{*}}, a Q_{i_{0}^{*}}\right)$ and $\left(Q_{i_{0}^{*}}, Q_{i_{1}^{*}}, a Q_{i_{1}^{*}}\right)$ are identical, i.e. the advantage is zero even if the adversary is computationally unbounded. We therefore have:

$$
\operatorname{Pr}[\text { Game3 } \Rightarrow 1]-\frac{1}{2}=0 .
$$

Combining all of the above:

$$
\begin{aligned}
& \operatorname{Adv}_{\Pi}^{\text {unlink }}(\mathcal{A}) \\
= & \operatorname{Pr}[\operatorname{Game} 0 \Rightarrow 1]-\frac{1}{2} \\
\leq & n_{C}^{2} \cdot\left(\operatorname{Pr}[\text { Game1 } \Rightarrow 1]-\frac{1}{2}\right) \\
\leq & n_{C}^{2} \cdot\left(\operatorname{Pr}[\operatorname{Game} \Rightarrow 1]-\frac{1}{2}+\mathbf{A d v}_{\pi}^{\text {wKSec}}\left(\mathcal{B}^{\prime}\right)\right) \\
\leq & n_{C}^{2} \cdot\left(\operatorname{Adv}_{\mathrm{AE}}^{\text {indsfcca }}(\mathcal{C})+\mathbf{A d v}_{\pi}^{\mathrm{wKSec}}\left(\mathcal{B}^{\prime}\right)\right) \\
\leq & n_{C}^{2} \cdot\left(\operatorname{Adv}_{\mathrm{AE}}^{\text {indsfcca }}(\mathcal{C})+n_{C} \cdot(1-1 /|h|) \cdot \mathbf{A d v}_{E\left(\mathbb{F}_{p}\right)}^{\mathrm{Gap}-\mathrm{DH}}(\mathcal{B})\right) .
\end{aligned}
$$

We note that if we were permitted to have $a$ small (as would be the case in the original EMV proposal) distinguishing the two distributions $\left(Q_{i_{0}^{*}}, Q_{i_{1}^{*}}, a Q_{i_{0}^{*}}\right)$ and $\left(Q_{i_{0}^{*}}, Q_{i_{1}^{*}}, a Q_{i_{1}^{*}}\right)$ may no longer be hard. Let $l$ denote the maximum bit length of $a$. The real question of interest would then be how small can $l$ be before the above problem becomes easy for computationally bounded adversaries. It is clear that the best attack against the problem for $2^{l} \ll q$ will be Pollard Lambda method [19], which runs in time $O\left(2^{l / 2}\right)$. This implies that a 32-bit randomizer $a$ only gives 16 bits of security and an 80-bit randomizer only gives 40 bits of security. 